\title{
ARTICLE
}

\section{Gln40 deamidation blocks structural reconfiguration and activation of SCF ubiquitin ligase complex by Nedd8}

\author{
Clinton $\mathrm{Yu}^{1, \star}$, Haibin Mao ${ }^{2, \star}$, Eric J. Novitsky ${ }^{3}$, Xiaobo Tang $^{2}$, Scott D. Rychnovsky ${ }^{3}$, Ning Zheng ${ }^{2}$ \& Lan Huang ${ }^{1}$
}

The full enzymatic activity of the cullin-RING ubiquitin ligases (CRLs) requires a ubiquitin-like protein (that is, Nedd8) modification. By deamidating Gln40 of Nedd8 to glutamate (Q4OE), the bacterial cycle-inhibiting factor (Cif) family is able to inhibit CRL E3 activities, thereby interfering with cellular functions. Despite extensive structural studies on CRLs, the molecular mechanism by which Nedd8 GIn40 deamidation affects CRL functions remains unclear. We apply a new quantitative cross-linking mass spectrometry approach to characterize three different types of full-length human Cul1-Rbx1 complexes and uncover major Nedd8-induced structural rearrangements of the CRL1 catalytic core. More importantly, we find that those changes are not induced by Nedd8(Q4OE) conjugation, indicating that the subtle change of a single Nedd8 amino acid is sufficient to revert the structure of the CRL catalytic core back to its unmodified form. Our results provide new insights into how neddylation regulates the conformation and activity of CRLs.

\footnotetext{
${ }^{1}$ Department of Physiology and Biophysics, University of California, Irvine, California 92697, USA. ${ }^{2}$ Department of Pharmacology and Howard Hughes Medical Institute, University of Washington, Seattle, Washington 98195, USA. ${ }^{3}$ Department of Chemistry, University of California, Irvine, California 92697 , USA. * These authors contributed equally to this work. Correspondence and requests for materials should be addressed to N.Z. (email: nzheng@uw.edu) or to L.H. (email: lanhuang@uci.edu).
} 
C ullin-RING ubiquitin ligases (CRLs) represent a superfamily of multi-subunit E3 ubiquitin ligases comprised of a cullin-RING catalytic core and adaptor proteins that mediate the recruitment of protein substrates ${ }^{1-6}$. Eight cullin family proteins (Cul1, Cul2, Cul3, Cul4A/B, Cul5, Cul7, Cul9 and APC2) are found in humans, each functioning as a scaffold on which a variety of CRLs are assembled. The SCF/CRL1 (Skp1-Cul1-F-box protein) complex represents the prototypical CRL E3, which uses Cul1-Rbx1 as the catalytic core ${ }^{2,7,8}$. The Cul1 scaffold binds the Skp1 adaptor and the Rbx1 RING subunit at its $\mathrm{N}$-terminal and C-terminal domains, respectively. Skp1 in turn docks F-box proteins, which are substrate receptors that confer substrate specificity to the SCF, while the RING-finger domain of Rbx1 engages ubiquitin-charged E2, mediating the transfer of ubiquitin to the F-box protein-bound substrate. A reconstructed structure model of the SCF based on crystal structures of several overlapping sub-complexes reveals an elongated E3 platform, in which the F-box protein is separated from the Rbx1-bound E2 by a $\sim 50-\AA$ distance 9 .

Covalent conjugation of ubiquitin-like protein Nedd8 (that is, neddylation) to a specific Lysine (Lys720) of Cul1 has been shown to promote both E2 recruitment and subsequent ubiquitin transfer, thereby stimulating the E3 activity of SCF ligases, ${ }^{2,10-13}$. Although the intact neddylated Cul1-Rbxl complex remains recalcitrant to crystallization, crystal structures of a truncated C-terminal domain of Cul5 in complex with Rbx1 have shed light on the effects of neddylation on the conformation of the cullin-RING catalytic core ${ }^{14}$. In the unneddylated form, the Cul5 ${ }^{\text {CTD }}$-Rbxl complex adopts a 'closed' conformation in which the RING-finger domain of Rbxl is nestled within a hydrophobic pocket of Cul5 ${ }^{\mathrm{CTD}}$. Upon neddylation, the RING-finger domain of Rbx1 is released from the pocket, deemed the 'open' state, but remains tethered by its $\mathrm{N}$-terminus to $\mathrm{Cul} 5$, presumably allowing the extended RING-finger to sample the three-dimensional (3D) space around Cul5. This conferred flexibility has been proposed to enable Rbx1 to close the distance between substrate and E2, facilitating the transfer of ubiquitin from E2 to substrate protein.

Notably, the cycle-inhibiting factors (Cifs) found in many pathogenic Gram-negative bacteria can irreversibly deamidate a specific glutamine residue $(\mathrm{Gln} 40)$ of Nedd8 and convert it to glutamate $^{15}$. This Q40E modification has no effect on cullin neddylation, but can effectively abolish the E3 activity of CRLs and affect proper cullin deneddylation by the COP9 signalosome $e^{15-18}$. These observations raise an intriguing question as to how the subtle change of a single Nedd 8 amino acid is able to negate the effect of neddylation in remodelling the $\sim 100-\mathrm{kDa}$ CRL catalytic core. In the structure of the neddylated Cul5 ${ }^{\mathrm{CTD}}$ Rbx1 complex, Gln40 of Nedd8 is close to the isopeptide bond between Nedd 8 and Cul5 and partially sandwiched between the two proteins. The amide group in the Gln40 side chain, however, is exposed to the solvent and does not participate in any hydrogen bond interactions ${ }^{14}$. The molecular mechanism by which Nedd8 Gln40 deamidation alters CRL functions remains elusive.

Recently, cross-linking mass spectrometry (XL-MS) has risen as a powerful method to study protein-protein interactions and characterize the structure of large protein complexes ${ }^{19-28}$. In comparison with X-ray crystallography or NMR, XL-MS approaches have much less restriction on sample preparation due to its sensitivity, flexibility and versatility, and are capable of capturing the dynamic states of large, heterogeneous protein structures. By stabilizing transient interactions, chemical crosslinking preserves various structural states of dynamic complexes, yielding a representation that describes the average state of a protein complex and providing a complementary set of structural data different from that obtained from rigid state data analyses such as X-ray crystallography. Recently, we have developed a new class of cross-linkers, that is, sulfoxide-containing MS-cleavable cross-linking reagents, to enable simplified and unambiguous identification of cross-linked peptides using multistage tandem mass spectrometry $\left(\mathrm{MS}^{n}\right)^{29-31}$. These new types of cross-linkers are robust and reliable, and have been successfully applied to define protein-protein interactions both in vitro $22,29,30$ and in $v_{i v o}{ }^{30}$. To establish a robust quantitative XL-MS (QXL-MS) platform to study dynamic protein complexes, we have then developed a pair of stable isotope-labelled amine reactive crosslinkers (that is, $\mathrm{d}_{0^{-}}$and $\mathrm{d}_{10^{-}}$-labelled dimethyl-disuccinimidyl sulfoxide (DMDSSO)), which allow simultaneous identification and quantitation of cross-linked peptides ${ }^{31}$. In combination with quantitative analysis, XL-MS can determine dynamic conversion between the average states of protein complexes under different conditions.

Here we employ this DMDSSO-based QXL-MS strategy to define the structural changes of full-length Cul1-Rbx1 modified by either wild-type Nedd8 or its Q40E mutant, which is the product of Gln40 deamidation. Quantitative similarities and differences in cross-linked peptide abundances can be attributed to the changes in protein complex structures under different conditions, as the occurrences of spatially proximal amino-acid residues suited for cross-linking is directly dependent on the $3 \mathrm{D}$ structural conformation of these complexes. Our results have provided new insights on how Nedd 8 modification impacts the topology of Cul1-Rbx1 and the effect of Nedd8 Gln40 deamidation on the structure of the activated CRL core.

\section{Results}

Reconstitution of SCF E3 activity with intact proteins. To enhance the solubility and stability of Cul1, we removed two short segments (see details in Methods) of Cull which were not visible in the crystal structure of Cul1-Rbxl complex (PDB: $1 \mathrm{LDJ})^{32}$, drastically improving protein behaviour. This truncated Cull and $\mathrm{Rbxl}^{16-108}$ were co-expressed and purified from Escherichia coli. The purified Cul1-Rbx1 was conjugated to wild-type Nedd8 to yield neddylated Cul1-Rbx1 complex (Nedd8 Cul1-Rbx1). The Q40E mutant Nedd8, in which Gln40 was replaced with Glu40 to mimic deamidated Nedd8, can also be efficiently conjugated to Cul1-Rbx1 to form Nedd8(Q40E) Cul1-Rbx1. Both neddylated Cul1-Rbx1 samples were purified with affinity-tagged Nedd8 after neddylation reaction to remove the unmodified species.

To understand ubiquitin ligase activities of different forms of Cul1-Rbx1, we have employed two in vitro ubiquitination assays: free ubiquitin chain assembly and ubiquitination of cryptochrome 2 (CRY2). CRY2, a key regulator of circadian rhythm, is a well-characterized substrate of the $\mathrm{SCF}^{\mathrm{FBXL} 3}$ ubiquitin ligase ${ }^{33-36}$. In both assays, we used Cdc34, the canonical E2 of Cul1. Consistent with previous reports, Cul1-Rbx1 can promote substrate-independent free ubiquitin chain assembly, while Nedd8 Cul1-Rbx1 significantly enhanced the reaction kinetics (Fig. 1a) $)^{37}$. In contrast, Nedd8(Q40E) $\sim$ Cul1-Rbx1 only exhibited comparable activity to unneddylated Cul1-Rbx1, which was much weaker than that of Nedd $8 \sim$ Cul1-Rbx1. This is consistent with the discovery that deamidation of Q40 in Nedd 8 abolishes the ligase activity of neddylated Cul1-Rbx1 (refs 15,16).

We further confirmed this observation with an in vitro ubiquitination assay of CRY2. As shown in Fig. 1b, polyubiquitin chains were formed on CRY2 in the presence of Nedd8 Cul1-Rbx1. In contrast, neither unneddylated nor Nedd8(Q40E)modified Cul1-Rbx1 complexes were able to catalyse ubiquitination of CRY2. This further confirms that Nedd8 Cul1-Rbx1 is the only active form and that deamidation of Q40 in Nedd8 can 
a

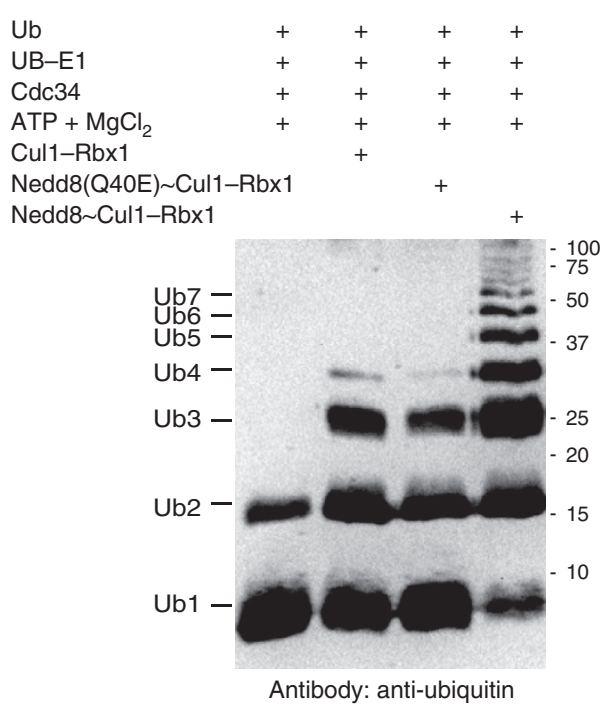

b

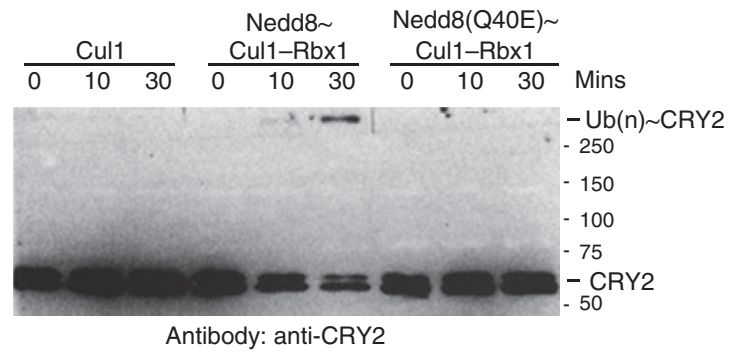

C

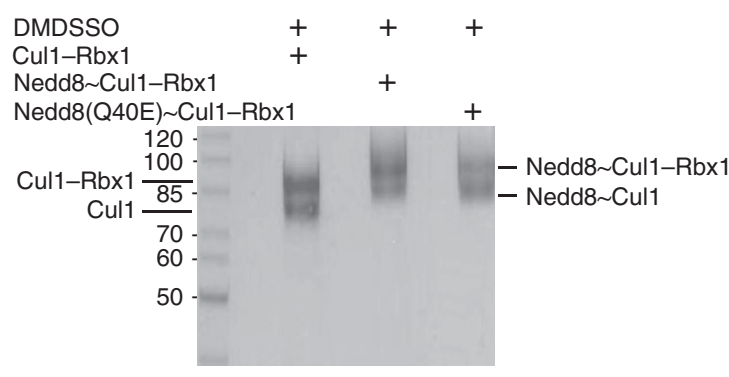

d

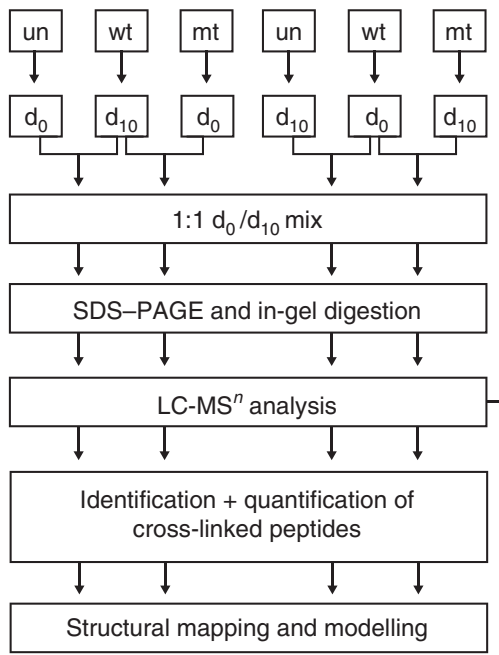

e

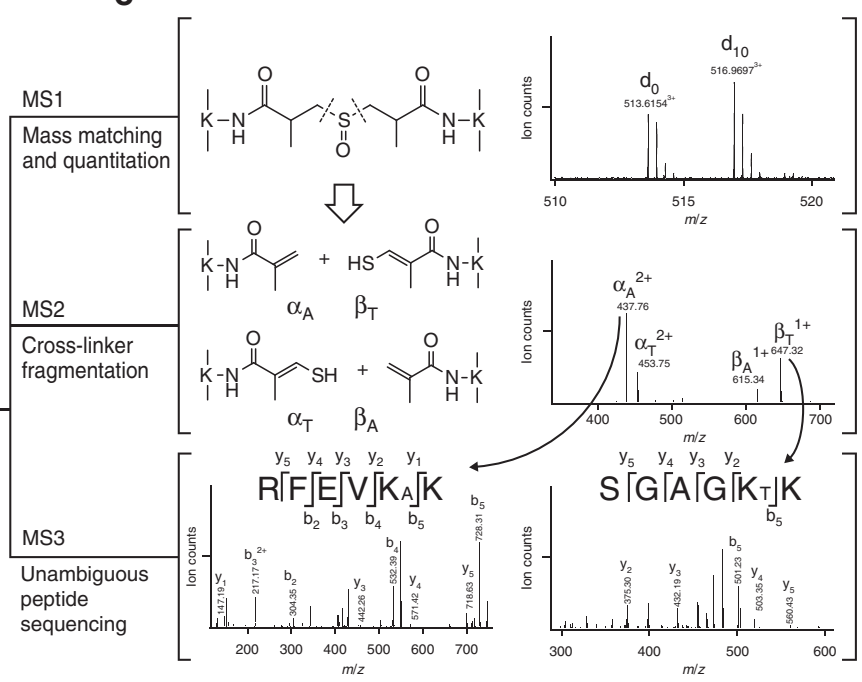

Figure 1 | Biochemical assays for ubiquitin ligase activity and general quantitative XL-MS experimental workflow. (a) Comparisons of ubiquitin ligase activities of different Cul1-Rbx1 variants on free ubiquitin chain assembly. Synthesized unanchored polyubiquitin chains were detected by anti-ubiquitin western blot. Highly efficient ubiquitin synthesis was only detected in the presence of Nedd8-Cul1-Rbx1 (lane 4). For visual clarity, ubiquitin polymers are simply abbreviated as $\mathrm{Ub}(\mathrm{n})$ (for example, Ub2 as ubiquitin dimer, Ub3 as ubiquitin trimer and so on). (b) Comparisons of ubiquitin ligase activities of different Cul1-Rbx1 variants on CRY2 ubiquitination. Ubiquitination reactions were quenched at indicated time points. Ubiquitinated CRY2 was detected using an anti-CRY2 antibody. Successful ubiquitination of CRY2 occurs only in the presence of Nedd8-Cul1-Rbx1 (lane 6). (c) SDS-PAGE analysis of DMDSSO cross-linked Cul1-Rbx1, Nedd8 Cul1-Rbx1 and Nedd8(Q4OE) Cul1-Rbx1 complexes. (d) do/d $\mathrm{d}_{10}$-DMDSSO-based quantitative XL-MS workflow for identifying and quantifying cross-linked peptides of Cul1-Rbx1 complexes. The three types of Cul1-Rbx1 complexes, that is, un, unneddylated; wt, wildtype neddylated; mt, mutant Q40E-neddylated, were first cross-linked by DMDSSO separately, two of which were then selected for mixing before SDS-PAGE. Four types of mixing were made to obtain sufficient pairwise comparison among the three samples. Gel bands representing cross-linked protein complexes were subsequently excised and in-gel digested before LC-MS ${ }^{n}$ analysis for identification and quantification. (e) Representative MS ${ }^{n}$ analysis of DMDSSO interlinked peptides. MS ${ }^{1}$ spectrum shows the detection of a pair of $\mathrm{d}_{0}$-DMDSSO and $\mathrm{d}_{10}$-DMDSSO cross-linked peptides $\left(\mathrm{m} / z 513.6154^{3}+\right.$

and $\mathrm{m} / z 516.9697^{3+}$ ), whose spectral relative abundance ratio is used for quantitation. $\mathrm{MS}^{2}$ analysis of the $\mathrm{d}_{0}$-DMDSSO interlinked peptides $\alpha-\beta$ ( $m / z$ $\left.513.6154^{3+}\right)$ yielded two peptide fragment pairs: $\alpha_{A} / \beta_{T}\left(m / z 437.76^{2+} / 647.32^{1+}\right)$ and $\alpha_{T} / \beta_{A}\left(m / z 453.75^{2+} / 615.34^{1+}\right)$, confirming its cross-link type as an interlink. Subsequent $M S^{3}$ analyses of the $\alpha_{A}\left(m / z 437.76^{2+}\right)$ and $\beta_{T}\left(647.32^{1+}\right)$ ions produced series of $y$ and $b$ ions that enabled unambiguous identification of $\alpha_{A}$ as RFEVK ${ }_{A} K$ of Rbx1 and $\beta_{T}$ as SGAGK ${ }_{T} K$ of Rbx1. Integration of the MS ${ }^{n}$ (that is, MS ${ }^{1}, M^{2}$ and MS ${ }^{3}$ ) data has confirmed the $\mathrm{d}_{0}$-DMDSSO cross-linked peptide as an intrasubunit interlink between $\mathrm{K} 19$ and $\mathrm{K} 25$ of Rbx1. $\mathrm{K}_{\mathrm{A}}$, alkene modified lysine; $\mathrm{K}_{\mathrm{T}}$, unsaturated thiol modified lysine.

in fact abrogate its activity. Compared with the free ubiquitin chain assembly assay, the polyubiquitin chain synthesis on CRY2 was highly processive, as the ubiquitinated CRY2 band was observed at the top of the gel as shown by western blot analysis.
Taken together, our results have demonstrated neddylation is essential for the activation of the Cul1-Rbx1 complex in protein ubiquitination. Importantly, distinct functional disparity between Nedd8 Cul1-Rbx1 and Nedd8(Q40E) Cul1-Rbx1 have been 
further validated, and only the former is the active E3 ligase for protein ubiquitination.

QXL-MS strategy. To understand molecular details underlying the functional differences between different forms of Cul1-Rbx1 complexes, we have employed a QXL-MS strategy based on a newly developed pair of stable isotope-coded MS-cleavable cross-linkers, $\mathrm{d}_{0}$-DMDSSO and $\mathrm{d}_{10}$-DMDSSO ${ }^{31}$ (Supplementary Fig. 1a,b), to examine the structural similarities and dissimilarities between these complexes. Concurrent usage of these two crosslinking reagents enables quantitative comparisons between the $3 \mathrm{D}$ structures of protein complexes under various conditions. To establish the QXL-MS workflow for comprehensive structural comparisons among unneddylated (un), wild-type neddylated (wt) and Q40E mutant neddylated (mt) Cul1-Rbx1 complexes, cross-linking conditions were first optimized through in vitro cross-linking of the three protein complexes with various concentrations of either $\mathrm{d}_{0}$-DMDSSO or $\mathrm{d}_{10}$-DMDSSO for different amounts of reaction times. Cross-linking efficiency was then evaluated by separating the resulting cross-linked products using one-dimensional SDS-polyacrylamide gel electrophoresis (SDS-PAGE). As shown in Fig. 1c, the resulting cross-linked products correspond well to respective molecular weights of these three complexes with $\sim 50 \%$ cross-linking efficiency. In addition, we have determined that $\mathrm{d}_{0^{-}}$and $\mathrm{d}_{10^{-}}$ DMDSSO reacted with Cul1-Rbxl complexes with similar efficiency as illustrated in Supplementary Fig. 1c, also reflected in previous testing on standard proteins ${ }^{31}$. These results demonstrate that $\mathrm{d}_{0^{-}}$and $\mathrm{d}_{10}$-labelled DMDSSO are well suited for QXL-MS analysis of these protein complexes.

To enable sufficient comparisons among the three different types of protein complexes with the minimal number of samples for analysis, we have strategically selected Nedd $8 \sim$ Cul1-Rbx1 as the cross-sample reference in the pairwise comparison experiments. As illustrated in Fig. $1 \mathrm{~d}, \mathrm{~d}_{10}$-DMDSSO cross-linked Nedd8 Cul1-Rbx1 was mixed with $\mathrm{d}_{0}$-DMDSSO cross-linked Cul1-Rbx1 or $\mathrm{d}_{0}$-DMDSSO cross-linked Nedd8(Q40E) Cul1Rbx1, followed by SDS-PAGE separation. The regions corresponding to expected cross-linked complexes were in-gel digested and the resulting peptides were subjected to liquid chromatography (LC)-MS ${ }^{n}$ analysis. DMDSSO cross-linked peptides were identified unambiguously based on $\mathrm{MS}^{n}$ data, that is, $\mathrm{MS}^{1}, \mathrm{MS}^{2}$ and $\mathrm{MS}^{3}$, as previously described ${ }^{29-31}$. Representative $\mathrm{MS}^{n}$ analyses of $\mathrm{d}_{0}$-DMDSSO and $\mathrm{d}_{10}$-DMDSSO interlinked peptides $\alpha-\beta\left(m / z 513.6154^{3+}\right.$ and $m / z 516.9697^{3+}$, respectively) are shown (Fig. 1e; Supplementary Fig. 2). As shown in Fig. 1e, $\mathrm{MS}^{2}$ analysis of the $\mathrm{d}_{0}$-DMDSSO interlinked peptide $\alpha-\beta$ yielded two expected fragment pairs $\alpha_{\mathrm{A}} / \beta_{\mathrm{T}}\left(\mathrm{m} / z 437.76^{2+} / 647.32^{1+}\right)$ and $\alpha_{\mathrm{T}} / \beta_{\mathrm{A}}\left(\mathrm{m} / \mathrm{z} 453.75^{2+} / 615.34^{1+}\right)$, which are characteristic of DMDSSO interlinked peptides ${ }^{31}$, confirming the type of cross-link observed here. Subsequent $\mathrm{MS}^{3}$ analysis of the $\alpha_{\mathrm{A}}$ fragment $\left(\mathrm{m} / z 437.76^{2+}\right)$ produced a series of $\mathrm{y}$ and $\mathrm{b}$ ions that enabled its unambiguous identification as ${ }^{21} \mathrm{RFEVK}_{\mathrm{A}} \mathrm{K}^{26}$ of Rbx1 with K25 modified with DMDSSO alkene remnant. Similarly, $\mathrm{MS}^{3}$ analysis of the $\beta_{\mathrm{T}}$ fragment $\left(\mathrm{m} / z 647.32^{1+}\right)$ identified its sequence unambiguously as ${ }^{15} \mathrm{SGAGK}_{\mathrm{T}} \mathrm{K}^{20}$ of Rbx1 with $\mathrm{K} 19$ modified with the unsaturated thiol remnant. Together with $\mathrm{MS}^{1}$ mass matching, we confidently determined this $\mathrm{d}_{0}$-DMDSSO cross-linked peptide as an intraprotein interlink between K19 and K25 of Rbx1. Similar MS ${ }^{n}$ analysis of the same peptide crosslinked with $\mathrm{d}_{10^{-}}$-DMDSSO $\left(\mathrm{m} / \mathrm{z} 516.9697^{3+}\right)$ further confirms and identifies the intrasubunit $\mathrm{K}-\mathrm{K}$ linkage within $\mathrm{Rbx} 1$ (Supplementary Fig. 2). On the basis of the identical fragmentation patterns of $\mathrm{d}_{0^{-}}$and $\mathrm{d}_{10^{-}}$DMDSSO cross-linked peptides, our results demonstrate that $\mathrm{d}_{0^{-}}$and $\mathrm{d}_{10^{-}}$DMDSSO contain the same functionality and characteristics required for the unambiguous identification of their respective cross-linked peptides by $\mathrm{MS}^{n}$ analysis. Therefore, identification of either of the $\mathrm{d}_{0^{-}}$or $\mathrm{d}_{10^{-}}$cross-linked peptide pairs in each pairwise experiment would allow us to quantify differences in their relative abundances.

To quantify the identified $\mathrm{d}_{0^{-}}$and $\mathrm{d}_{10}$-DMDSSO cross-linked peptides, we then determined the relative abundance ratios of corresponding peptide pairs based on their $\mathrm{MS}^{1}$ spectral intensities. The same pairwise comparison experiments were repeated using reversed cross-linker treatments (that is, $\mathrm{d}_{0}$-DMDSSO cross-linked Nedd8 Cul1-Rbx1 was mixed with $\mathrm{d}_{10}$-DMDSSO cross-linked Cul1-Rbx1 or $\mathrm{d}_{10}$-DMDSSO crosslinked Nedd8(Q40E) Cul1-Rbx1) to rule out cross-linking bias due to reagent deuteration (Fig. 1d).

Mapping XL-MS data to Cul1-Rbxl complexes. The current structural model of unneddylated Cul1-Rbx1 is described in Fig. 2a, based on a previously reported crystal structure of full-length human Cul1-Rbx1 (PDB 1LDJ) ${ }^{32}$. In this structure, the N-terminal domain of Cull consists of three helical repeats (repeat 1, 2 and 3), each comprising five $\alpha$-helices. These three repeats pack consecutively to form a long stalk-like shape. The Cul1 C-terminal domain (CTD) is composed of a four-helix bundle (4HB), an $\alpha / \beta$ domain and two copies of the winged-helix motif (WHA and WHB). The $4 \mathrm{HB}$ connects the N-terminal domain to CTD and organizes other subdomains in the CTD. It packs with the $\alpha / \beta$ domain and the long H29 helix, which connects WHA and WHB. The $\alpha / \beta$ domain and the $\mathrm{N}$-terminal $\beta$-strand of Rbx1 form an intermolecular five-stranded $\beta$-sheet. One face of the WHB interacts with the long H29 helix and the $4 \mathrm{HB}$, and the other contacts the RING domain of Rbxl. This compact architecture has been proposed to represent the 'closed' conformation of the Cull-Rbxl complex (Fig. 2a) ${ }^{14}$.

Although there is no high-resolution structure available for the Nedd $8 \sim \mathrm{Cul} 1-\mathrm{Rbx} 1$ complex, the crystal structure of Nedd $8 \sim \mathrm{Cull}^{\mathrm{CTD}}-\mathrm{Rbx} 1$ was previously resolved ${ }^{14}$. By threading the Cul1 ${ }^{\text {CTD }}$ sequence into the neddylated Cul5 structure, we have derived a homology model of Nedd8 Cul1-Rbx1 (Fig. 2b). Similar to the structure of Nedd $8 \sim \mathrm{Cul} 5^{\mathrm{CTD}}-\mathrm{Rbx} 1$ (ref. 14 ), this model shows that neddylation has minor effects on the structures of individual subdomains, but induces marked rearrangements in their relative positions. The $\mathrm{H} 29$ helix rotates about $45^{\circ}$, which changes the $\mathrm{WHB}$ position relative to the $4 \mathrm{HB}$ and $\alpha / \beta$ domain. The repositioning of WHB abolishes the interaction between the WHB and the Rbx1 RING domain and frees the latter from the Cul1 scaffold. Nedd8 contacts the WHB to stabilize this 'open' state of the Cul1-Rbx1 complex. Two orientations for the RING, resulting from crystal packing, are observed in the crystal structure of Nedd $8 \sim \mathrm{Cul} 5{ }^{\mathrm{CTD}}-\mathrm{Rbx} 1$, indicating that the relative position of the RING domain and cullin scaffold are very promiscuous in solution. Therefore, we have generated two structural models of Nedd8 Cul1-Rbx1 to describe the two different RING conformations (I and II) in the complex, and their overlays are illustrated in Fig. 2b. As shown, RING I (in yellow) is more proximal to the Cull scaffold, whereas RING II (in grey) is more distal. It is noted that the orientations of the Cull scaffold and Nedd 8 remain the same in both RING conformations (Fig. 2b).

To further elucidate the structures of Cul1-Rbx1 complexes, we focused on the identification and quantification of interlinked peptides as they are most informative in describing residue proximity and interaction contacts in $3 \mathrm{D}$ structures. With our XL-MS strategy, we have identified a total of 68 unique interlinked $\mathrm{d}_{0} / \mathrm{d}_{10}$ peptide pairs from eight replicate sets 
a

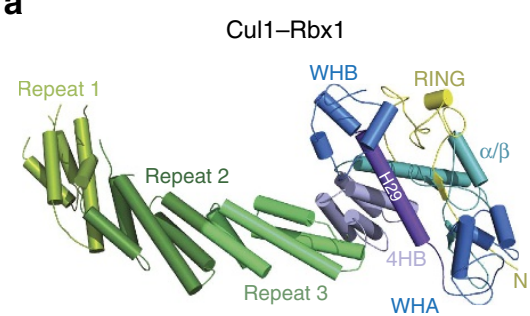

C

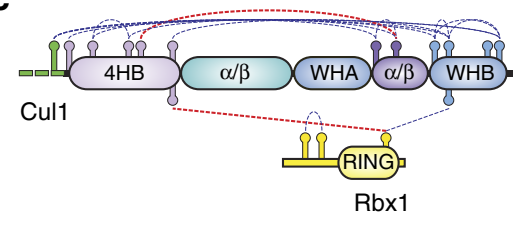

b

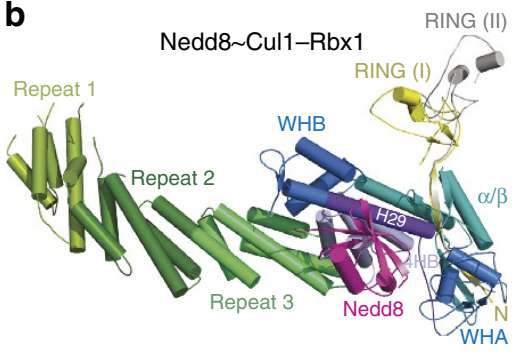

d

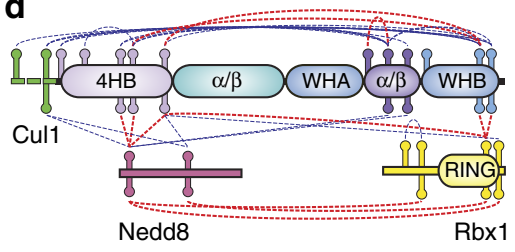

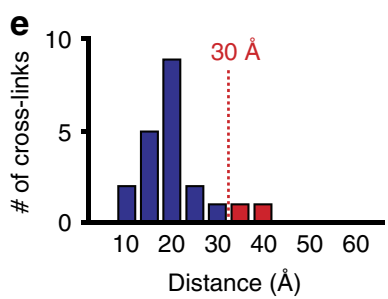
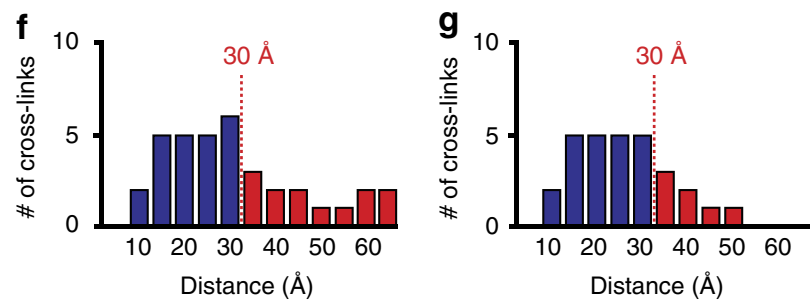

Figure 2 | Mapping cross-link data onto current structural models of Cul1-Rbx1 complexes. (a) The known structure of unneddylated Cul1-Rbx1 complex. (b) The overlay of the two homology models of neddylated Cul1-Rbx1 derived from Nedd8 Cul5CTD -Rbx1 structure, depicting two conformations of the Rbx1 RING domain with I in yellow and II in grey. On the basis of the identified interlinked peptides, the cross-link maps were generated for (c) Cul1-Rbx1 and (d) Nedd8 Cul1-Rbx1 complexes. Note: linkages between residues with spatial distances below $30 \AA$ are shown in bluedotted lines, while those above $30 \AA$ in red-dotted lines, correlating with colour-coded bar graphs in $\mathbf{e}-\mathbf{g}$. (e) The distribution plot of identified linkages versus their spatial distances between interlinked lysines in Cul1-Rbx1 structure. (f) The distribution plot of identified linkages versus their spatial distances between interlinked lysines in Nedd8 Cul1-Rbx1 structure models. (g) The distribution plot of identified linkages involving only Cul1 and Nedd8 versus their spatial distances between interlinked lysines in Nedd8 Cul1-Rbx1 structure models.

of comparison experiments (Supplementary Table 1), representing 27 intraprotein and 17 interprotein linkages. To correlate our XL-MS data with the current structural models of Cul1-Rbx1 complexes, we first generated K-K linkage maps of Cul1-Rbx1 and Nedd8 Cul1-Rbx1 based on the interlinks identified from each sample, as shown in Fig. $2 c$,d. It is important to note that our structural models of Nedd8 $\sim \mathrm{Cul}-$ Rbx1 with either RING (I) or RING (II) conformations have the same cross-link maps as shown in Fig. 2d. Interestingly, 23 of 25 intraprotein Cull K-K linkages are localized in Cul1 ${ }^{\mathrm{CTD}}$ regions that interact with Rbx1 and Nedd8. In addition, 5 and 10 linkages represent interprotein interactions of Cull with $\mathrm{Rbx} 1$ or Nedd8, respectively. Collectively, extensive interactions among the three proteins were detected for us to evaluate the structural differences between the various forms of Cul1-Rbx1 complexes.

Next, we have mapped the identified cross-linked residues onto the structural models of Cul1-Rbx1 and Nedd8 $\sim$ Cul1-Rbx1, respectively, and calculated the distances between $\alpha$-carbons $\left(\mathrm{C}_{\alpha}-\mathrm{C}_{\alpha}\right.$ distance $)$ of cross-linked lysines using the molecular visualization software PyMOL. Considering the lengths of the DMDSSO (11 $\AA$ ) and lysine side chains as well as backbone dynamics, the theoretical upper limit for the $\mathrm{C}_{\alpha}-\mathrm{C}_{\alpha}$ distance between DMDSSO cross-linked lysine residues is $\sim 30 \AA$, suggesting that lysines within distance $<30 \AA$ can be preferably cross-linked by DMDSSO. To examine the distance constraints of identified cross-links, we have plotted the distance distribution of the Cul1-Rbx1 cross-link data set (Fig. 2e). Ninety per cent of cross-links satisfy the distance cutoff of $30 \AA$, indicating a good correlation with the current known structure of Cul1-Rbx1. However, when plotting Nedd8 $\sim$ Cul1-Rbx1 cross-link data to either of our homology-derived models, only $64 \%$ of cross-links (23/36) are within the desired distance constraint (Fig. 2f). In fact, the cross-links outside the cutoff predominantly represent interactions among Nedd8, Rbx1 and the C-terminal domain of Cull. As Rbxl is suspected to be mobile in previous publications ${ }^{14}$, we then excluded 6 Rbx1-associated cross-links, thus yielding 30 remaining cross-links describing interactions within and between Nedd 8 and Cul1 proteins. As a result, $\sim 73 \%$ of linkages (22/30) fall within our expected distance constraints (Fig. 2g), with the 8 outliers all representing cross-links that involve either Nedd8 or the winged-helix domains of Cul1 1 CTD. This discrepancy could be explained by either the inaccuracy of the Nedd8 $\sim$ Cul1-Rbx1 structural model in those regions or a highly dynamic topology associated with the 'open' conformation.

Quantitation of DMDSSO cross-linked peptides. Generally, the likelihood of forming a cross-link between two given lysine residues is dependent on multiple factors. One of the important aspects is the 3D spatial distance between cross-linkable lysines. In addition, the relative orientations of proteins and their subdomains in different conformations under compared conditions can influence the relative reactivity of lysine residues. For instance, lysine residues localized in buried or protected regions would have decreased solvent and cross-linker accessibility compared with flexible, unprotected regions. Moreover, certain 
conformations could potentially influence the electronic environments of lysine residues by positioning them to form salt-bridge interactions with nearby acidic residues, decreasing their relative reactivity. Therefore, a combination of multiple factors could ultimately be responsible for the differences in observed spectral abundances of cross-linked peptides. Nonetheless, comparative analysis using QXL-MS strategies can unravel conformational changes of protein complexes under different conditions ${ }^{24,38}$. Of the total 68 unique interlinks identified in this work, 41 were identified at least in three biological replicates-our minimum requirement for reproducibility-representing 26 unique and high-confidence $\mathrm{K}-\mathrm{K}$ linkages that were used for quantitative structural comparisons. Among them, there are two linkages associated with K720 of Cull, which is the neddylation site and therefore covalently modified in the two neddylated Cul1-Rbx1 complexes, but free in unmodified Cul1-Rbxl complex. As such, the two identified interlinked peptides associated with K720 of Cul1 were only detected in Cul1-Rbx1 complex, and were excluded from further analyses. The final list of 24 unique and quantifiable $\mathrm{K}-\mathrm{K}$ linkages used for assessing structural changes of Cul1-Rbx1 complexes is summarized in Table 1 . As shown, 13 were intraprotein (12 Cul1-Cul1 and $1 \mathrm{Rbx} 1-\mathrm{Rbx} 1$ ) and 11 were interprotein (3 Cul1-Rbx1, 7 Cul1-Nedd8 and 1 Rbx1-Nedd8) interlinks. Among them, 15 linkages exhibited significant changes ( $\geq 4$-fold) in their relative abundances and suggested structural differences in different samples, while the remaining 9 displayed marginal changes $(<2$-fold $)$, indicating those interaction regions are relatively stable.
Comparison of Cul1-Rbxl and Nedd8 Cul1-Rbx1 complexes. Existing structural models have suggested that unneddylated Cul1-Rbx1 adopts a 'closed' conformation, while neddylated Cul1-Rbx1 exists in an 'open' state, as represented in Fig. 3a,b. To determine the structural effects of neddylation in the context of the full-length proteins, we examined intraprotein interlinks identified within Cull and Rbx1, respectively. For the 12 intraprotein interlinks identified within Cul1, 6 of them (that is, Cul1 $1^{\mathrm{K} 410-\mathrm{K} 743}$, Cul1 ${ }^{\mathrm{K} 417-\mathrm{K} 689}, \mathrm{Cul1}^{\mathrm{K} 468-\mathrm{K} 693}, \mathrm{Cul1}^{\mathrm{K} 472-\mathrm{K} 689}, \mathrm{Cul1}^{\mathrm{K} 472-\mathrm{K} 693}$ and Cul1 ${ }^{\mathrm{K} 701-\mathrm{K} 708}$ ) exhibited below twofold difference between Cul1-Rbx1 and Nedd8 Cul1-Rbx1 complexes, suggesting that there were no substantial structural reorientations between these cross-linked lysine residues upon neddylation (Table 1). In consistence with the cross-linking data, all of their $\mathrm{C}_{\alpha}-\mathrm{C}_{\alpha}$ distances are within $30 \AA$ in the current Cul1-Rbx1 and Nedd $8 \sim \mathrm{Cul} 1-\mathrm{Rbx} 1$ complex models. For example, the relative spectral abundance ratio of the $\mathrm{Cul1}^{\mathrm{K} 472-\mathrm{K} 693}$ linkage in unneddylated and neddylated Cul1-Rbx1 is $\sim 1$, and their respective $\mathrm{C}_{\alpha}-\mathrm{C}_{\alpha}$ distances are 16.0 and $16.4 \AA$ (Fig. $3 \mathrm{c}$ ). Although the $4 \mathrm{HB}$ and $\alpha / \beta$ domains containing these two residues become closer (Fig. 3a,b), the overall $3 \mathrm{D}$ spatial distance of these two residues has minimal change, thus leading to comparable cross-linking efficiency.

In contrast, the remaining six intraprotein interlinks of Cul1 had at least fourfold difference in their relative abundance ratios, in which five interlinks (that is, Cul1 ${ }^{\mathrm{K} 337-\mathrm{K} 750}, \mathrm{Cul1}{ }^{\mathrm{K} 410-\mathrm{K} 750}$, $\mathrm{Cul1}^{\mathrm{K} 464-\mathrm{K} 693}$, Cul1 ${ }^{\mathrm{K} 464-\mathrm{K} 743}$ and $\mathrm{Cul1}^{\mathrm{K} 693-\mathrm{K} 743}$ ) were detected much more dominantly in Nedd $8 \sim$ Cul1-Rbxl complex and one (that is, Cul1 ${ }^{\mathrm{K} 431-\mathrm{K} 472}$ ) significantly abundant in unneddylated Cul1-Rbx1 (Table 1; Fig. 3). These differences indicate that the

Table 1 | Comparative linkage profiles for SCF core ligases as determined by LC-MS ${ }^{n}$.

Linkages identified

\begin{tabular}{|c|c|c|c|c|c|c|}
\hline Linkage & Cul1 & Cul1 & Rbx1 & Rbx1 & Nedd8 & Cul1-F \\
\hline \multirow[t]{13}{*}{ Cul1-Cul1 } & K337 & K750 & & & & 37. \\
\hline & K410 & K720 & & & & 38. \\
\hline & K410 & K743 & & & & 17. \\
\hline & K410 & K750 & & & & 24 \\
\hline & K417 & K689 & & & & 18 \\
\hline & K431 & K472 & & & & 9 \\
\hline & K464 & K693 & & & & 15 \\
\hline & K464 & K743 & & & & 15 \\
\hline & K468 & K693 & & & & 13. \\
\hline & K472 & K689 & & & & 16 \\
\hline & K472 & K693 & & & & 15 \\
\hline & K693 & K743 & & & & 24 \\
\hline & K701 & K708 & & & & 11 \\
\hline \multirow[t]{4}{*}{ Cul1-Rbx1 ${ }^{\dagger}$} & K493 & & K89 & & & 30. \\
\hline & K720 & & K89 & & & 11. \\
\hline & K743 & & K89 & & & 26 \\
\hline & K750 & & K89 & & & 22 \\
\hline Rbx1-Rbx1 & & & K19 & K25 & & 18. \\
\hline \multirow[t]{7}{*}{ Cul1-Nedd8 } & K410 & & & & K6 & \\
\hline & K464 & & & & K6 & \\
\hline & K468 & & & & K6 & \\
\hline & K493 & & & & K6 & \\
\hline & K493 & & & & K48 & \\
\hline & K693 & & & & K6 & \\
\hline & K701 & & & & K6 & \\
\hline Rbx $^{\dagger}$-Nedd8 & & & K89 & & $\mathrm{K} 48$ & \\
\hline
\end{tabular}

Mapped distances $(\AA)$

wtNedd8

$\sim$ Cul1-Rbx

26.5

21.7

22.5

21.0

24.8

9.3

11.9

26.3

12.2

10.7

16.4

26.6

10.5

40.8 (I)/55.2 (II)

72.8 (I)/82.8 (II)

59.2 (I)/66.0 (II)

54.5 (I)/64.9 (II)

---

17.0
30.6

35.3

41.0

29.9

28.7

19.6

65.0 (I)/74.3 (II)

\begin{tabular}{ccc}
\multicolumn{3}{c}{ Quantitative ratio $^{\star}$} \\
\hline Cul1- & $\begin{array}{c}\text { wtNedd8 } \\
\text { Rbx1 }\end{array}$ & $\begin{array}{c}\text { mtNedd8 } \\
\text { Cul1-Rbx1 }\end{array}$ \\
$\sim$ Cul1-Rbx1 \\
0.05 & 1.00 & 0.20 \\
1.00 & 0.01 & 0.01 \\
0.85 & 0.67 & 1.00 \\
0.14 & 1.00 & 0.36 \\
1.00 & 0.60 & 0.91 \\
1.00 & 0.24 & 0.50 \\
0.36 & 1.00 & 0.41 \\
0.05 & 1.00 & 0.22 \\
1.00 & 0.49 & 0.85 \\
1.00 & 0.87 & 0.90 \\
0.94 & 0.87 & 1.00 \\
0.03 & 1.00 & 0.17 \\
0.69 & 1.00 & 0.92 \\
1.00 & 0.67 & 0.77 \\
1.00 & 0.00 & 0.00 \\
0.06 & 1.00 & 0.10 \\
0.11 & 1.00 & 0.05 \\
1.00 & 0.62 & 0.71 \\
0.01 & 1.00 & 0.22 \\
0.01 & 1.00 & 0.12 \\
0.05 & 1.00 & 0.23 \\
0.04 & 0.07 & 1.00 \\
0.01 & 0.11 & 1.00 \\
0.00 & 1.00 & 0.11 \\
0.01 & 1.00 & 0.13 \\
0.02 & 0.96 & 1.00 \\
& &
\end{tabular}

Quantitative ratio*

0.01

1.00

0.60

0.24

1.00

0.49

0.87

1.00

1.00

0.00

1.00

0.62

1.00

0.07

0.11

1.00

0.96 


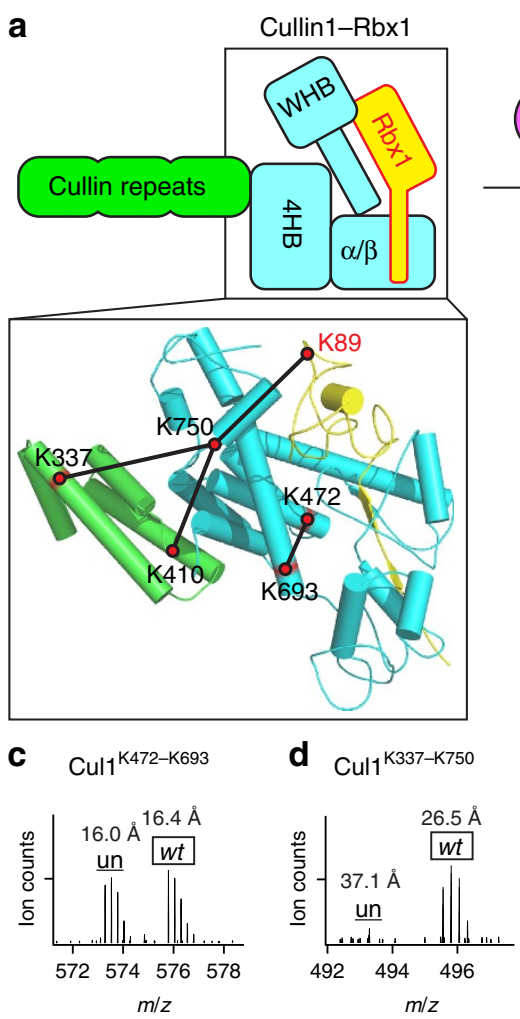

b
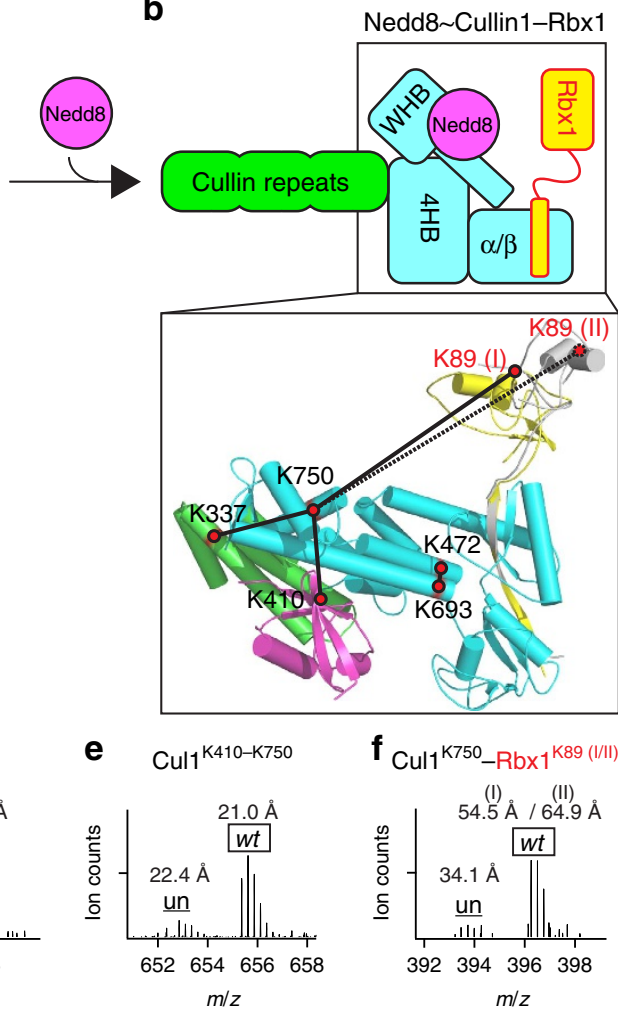

un : Cul1-Rbx1 $\quad$ wt : Nedd8 Cul1-Rbx1

Figure 3 | Quantitative analysis of K-K linkages to determine neddylation-dependent structural changes in the Cul1-Rbx1 complex. Structural representation of (a) unneddylated Cul1-Rbx1 in the 'closed' state, (b) neddylated Cul1-Rbx1 in the 'open' conformation, in which K89 (I) and K89 (II) represent K89 position in Rbx1 RING (I) (in yellow) or (II) (in grey) conformations, respectively. The insets display the mapping of four selected interlinks onto the structures of Cul1-Rbx1 complexes, whose $\mathrm{MS}^{1}$ spectra are displayed as follows: (c) Cul1 ${ }^{\mathrm{K} 472-\mathrm{K} 693}$, (d) Cul1 ${ }^{\mathrm{K} 337-\mathrm{K} 750}$; (e) Cul1 ${ }^{\mathrm{K} 410-\mathrm{K} 750 \text {; }}$ (f) $\mathrm{Cul1}{ }^{\mathrm{K} 750}-\mathrm{Rbx} 1^{\mathrm{K} 89(\mathrm{I} / \mathrm{II})}$. These $\mathrm{d}_{0} / \mathrm{d}_{10}$-DMDSSO cross-linked peptide pairs measured in $\mathrm{MS}^{1}$ were used to determine their relative abundance ratios between unneddylated and neddylated Cul1-Rbx1 complexes for quantitative analysis (Table 1).

two complexes feature substantial structural differences in regions containing the cross-linked lysines. In this study, two lysine residues that are proximal $(<30 \AA)$ would have higher chance of being captured by DMDSSO cross-linkers, which in turn increases the spectral abundance compared with one between lysine residues that are spatially distant $(>30 \AA)$. Figure $3 \mathrm{~d}$ displays the MS spectrum of the Cul1 ${ }^{\mathrm{K} 337-\mathrm{K} 750}$ interlink, and quantitative analysis revealed that this interaction occurs much more favourably in Nedd $8 \sim$ Cul1-Rbx1 than Cul1-Rbx1, on an average of 20:1. From the current models, the $C_{\alpha}-C_{\alpha}$ distances between K337 and K750 of Cull were calculated to be 36.3 and $26.5 \AA$ based on the Cul1-Rbxl structure and our Nedd $8 \sim$ Cul1Rbx 1 model, respectively. These calculated $\mathrm{C}_{\alpha}-\mathrm{C}_{\alpha}$ distances fall outside of and within the distance that can be cross-linked by DMDSSO, which are in agreement with the increased spectral abundance of this interlink in Nedd $8 \sim \mathrm{Cul} 1-\mathrm{Rbx} 1$ compared with Cul1-Rbx1.

Similarly, the intraprotein interlink Cul1 ${ }^{\mathrm{K} 410-\mathrm{K} 750}$ was calculated to be on an average of approximately seven times more abundant in wild-type neddylated than unneddylated forms (Table 1; Fig. 3e). However, distinct from the Cul1 ${ }^{\mathrm{K} 337-\mathrm{K} 750}$ interlink, the $\mathrm{C}_{\alpha}-\mathrm{C}_{\alpha}$ distances of $\mathrm{K} 410$ and K750 in the current models were determined to be 22.4 and $21.0 \AA$, respectively. Despite this similarity in their calculated proximities, the differential spectral abundance suggests that their cross-link is obstructed in unneddylated Cul1. In addition to distance, crosslinked peptide spectral abundance is influenced by the relative orientation of the lysine pair and their surroundings. In fact,
K410 and K750 of unneddylated Cul1 point away from each other and are separated by other residues, which can presumably impede the cross-linking reaction. Similarly, an additional four Cull intraprotein interlinks (that is, $\mathrm{Cull}^{\mathrm{K} 464-\mathrm{K} 693}$, $\mathrm{Cul1}^{\mathrm{K} 464-\mathrm{K} 743}$, Cul1 ${ }^{\mathrm{K} 693-\mathrm{K} 743}$ and $\mathrm{Cul1}^{\mathrm{K} 431-\mathrm{K} 472}$ ) have no apparent correlation between their relative spectral abundance ratios and respective $\mathrm{C}_{\alpha}-\mathrm{C}_{\alpha}$ distance $(<30 \AA)$. However, most of them can be rationalized based on the structural environment of the lysine residues in the context of the current structure models

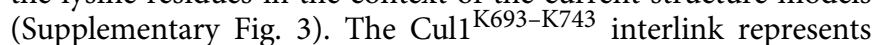
the only noticeable outlier, which is $>30$-folds more abundant in Nedd8 Cul1-Rbx1 than Cul1-Rbx1, albeit a similar $\mathrm{C}_{\alpha}-\mathrm{C}_{\alpha}$ distance in the two models. K693 and K743 are located on the H29 helix and the WHB domain, which together act as a single rigid body. Their preferred cross-links in the neddylated Cul1-Rbx1 cannot be explained without significant changes of the current model of Nedd $8 \sim$ Cul1-Rbx1. Overall, our results suggest that certain regions in the Cull scaffold, including the structural elements where those lysines are located, likely undergo profound structural reorientations in response to neddylation.

To further dissect the impact of neddylation, we examined the three unique Cul1-Rbx1 interprotein $\mathrm{K}-\mathrm{K}$ linkages identified here (Table 1; Figs 3 and 4). Two cross-links, Cul1 ${ }^{\mathrm{K} 743}-\mathrm{Rbx1}{ }^{\mathrm{K} 89}$ and $\mathrm{Cul1} 1^{\mathrm{K} 750}-\mathrm{Rbx} 1^{\mathrm{K} 89}$, were quantitatively determined to have spectral abundances on an average of 10-fold higher in neddylated Cul1-Rbxl compared with their unneddylated counterparts. Mapping of $\mathrm{Cul}^{\mathrm{K} 743}-\mathrm{Rbx}^{\mathrm{K} 89}$ and $\mathrm{Cul}^{\mathrm{K} 750}-\mathrm{Rbx}^{\mathrm{K} 89}$ to the Cul1-Rbx1 structure determines their 


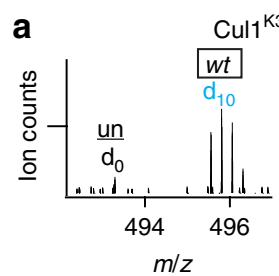

Cul1 ${ }^{\mathrm{K} 337-\mathrm{K} 750}$
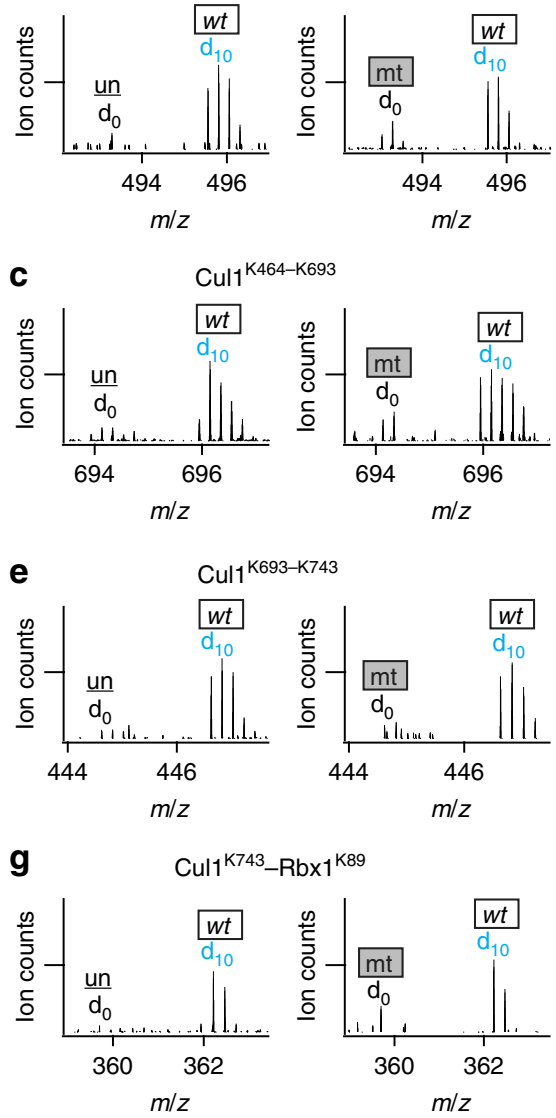
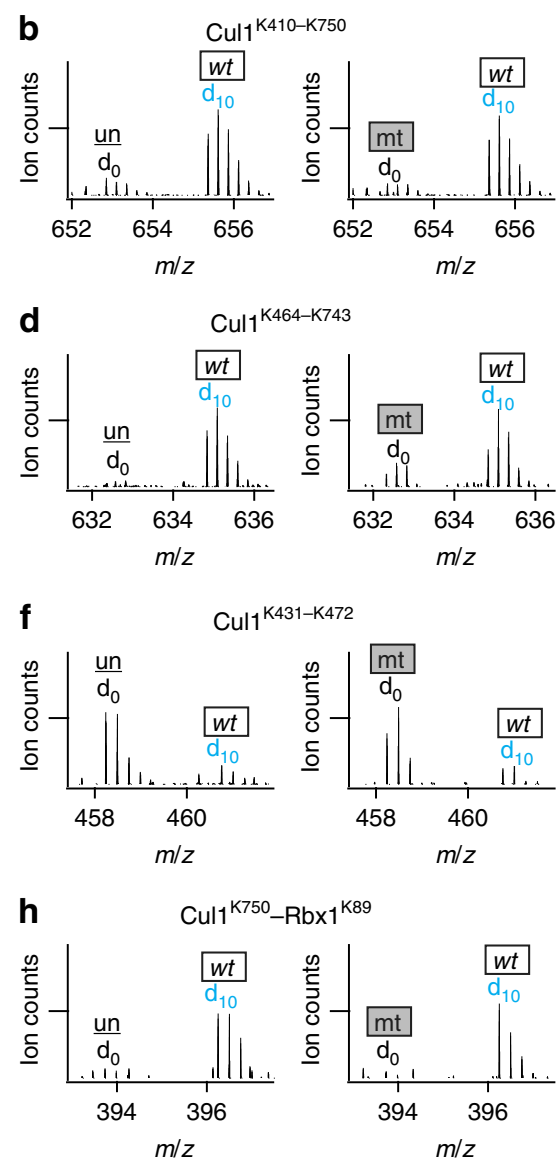

un : Cul1-Rbx1 wt : Nedd8 Cul1-Rbx1 $\mathrm{mt}$ : Nedd8(Q40E) Cul1-Rbx1

Figure 4 | Deciphering the structural dynamics of Cul1-Rbx1 complexes using QXL-MS. Eight selected K-K linkages are presented to describe conformational changes in the three types of Cul1-Rbx1 complexes. Two sets of pairwise comparison results (that is, un ( $\left.d_{0}\right)$ versus wt $\left(d_{10}\right)$ and mt $\left(d_{0}\right)$ versus wt $\left(d_{10}\right)$ ) are displayed for each selected cross-link, in which both the un and mt forms were cross-linked by $d_{0}$-DMDSSO and the wt form was cross-linked by $\mathrm{d}_{10}$-DMDSSO. MS ${ }^{1}$ spectra of (a) Cul1 K337-K750, (b) Cul1 K410-K750, (c) Cul1 K464-K693, (d) Cul1 K464-K743, (e) Cul1 K693-K743, (f) Cul1 ${ }^{\mathrm{K} 431-K 472}$, (g) Cul1 ${ }^{\mathrm{K} 743}-\mathrm{Rbx1} 1^{\mathrm{K} 89}$ and (h) Cul1 ${ }^{\mathrm{K} 750}-\mathrm{Rbx1} 1^{\mathrm{K} 89}$. The sequences of these cross-links are summarized in Supplementary Table 1. The relative spectral abundance of cross-links $\left(d_{0}: d_{10}\right)$ measured during MS analysis describes the cross-linkability of lysine residues in $3 D$ structure.

$\mathrm{C}_{\alpha}-\mathrm{C}_{\alpha}$ distances to be 31.9 and $34.1 \AA$, respectively, just outside the range covered by DMDSSO and accounting for their low cross-linking abundances. However, when mapped to our homology-derived models of neddylated Cul1-Rbx1 with either RING (I) or RING (II) conformations, those same interlinks yielded $\mathrm{C}_{\alpha}-\mathrm{C}_{\alpha}$ distances of $59.2 \AA$ (I)/66.0 $\AA$ (II) and $57.5 \AA$ (I) $/ 64.9 \AA$ (II), respectively (Table 1 ; Fig. $3 \mathrm{f}$ ), even more unlikely to be cross-linked by DMDSSO. Instead, these unusual cross-links must be explained by either the structural flexibility of the 'openstate' conformation exhibited by Nedd $8 \sim \mathrm{Cul} 1-\mathrm{Rbx} 1$ or a geometry different from the current model. In the crystal structure of $\mathrm{Nedd} 8 \sim \mathrm{Cul}^{\mathrm{CTD}}-\mathrm{Rbx} 1$, neddylation causes the globular RING domain of Rbx1 to eject from the WHB while remaining tethered to the Cul5 $5^{\mathrm{CTD}}$ by its $\mathrm{N}$-terminal sequence. As a result, Rbx1 is free to sample the $3 \mathrm{D}$ space above Cul5 ${ }^{\mathrm{CTD}}$. Our observations on the three Cul1-Rbx1 interprotein interlinks indicate that such a dynamic topology is plausible and can account for the formation of linkages with lysine residues that are too distant to be cross-linked in the unneddylated complex.

Interestingly, mapping of the identified K-K linkages between Cull and Nedd8 to the homology-derived Nedd8 Cul1-Rbx1 model shows that three out of seven cross-linking events were calculated to bridge $\mathrm{C}_{\alpha}-\mathrm{C}_{\alpha}$ distances $>30 \AA$, with two more above $28.5 \AA$ (Table 1). This suggests that the position of Nedd8 relative to $4 \mathrm{HB}$ and $\alpha / \beta$ subdomains of $\mathrm{Cul1}$, which comprise the majority of the Cul1-Nedd8 interlinks, may not be accurate in the current model. On one hand, the crystal structure of the Nedd $8 \sim$ Cul 5 CTD_Rbx1 complex, which our Nedd8 $\sim$ Cul1Rbx1 model was based on, might represent snapshots of an otherwise dynamic scaffold in addition to the flexibly linked Rbx1 RING domain. On the other hand, it remains possible that neddylation may cause different conformational changes on different cullins. Therefore, a more accurate structure model of the Nedd8 $\sim$ Cul1-Rbxl complex is needed to explain all comparative cross-links between the free and modified Cul1-Rbx1 assembly.

Effects of Nedd8 deamidation on Cul1-Rbx1. To investigate the structural mechanism underlying deamidation of Nedd8, we conducted pairwise structural comparisons between Nedd8 Cul1-Rbx1 (wt) and Nedd8(Q40E) Cul1-Rbx1 (mt) complexes using the same DMDSSO-based QXL-MS strategy as described above. Similar to the previous results obtained from the comparison between unneddylated and wild-type neddylated Cul1Rbx1 complexes, the six Cul1 interlinks (that is, Cul1 $1^{\mathrm{K} 410-\mathrm{K} 743}$, $\mathrm{Cul1}^{\mathrm{K} 417-\mathrm{K} 689}, \mathrm{Cul1}^{\mathrm{K} 468-\mathrm{K} 693}, \mathrm{Cul1}^{\mathrm{K} 472-\mathrm{K} 689}, \mathrm{Cul1}^{\mathrm{K} 472-\mathrm{K} 693}$ and $\mathrm{Cul1}{ }^{\mathrm{K} 701-\mathrm{K} 708}$ ) and one Rbx1 interlink (that is, $\mathrm{Rbx} 1^{\mathrm{K} 19-\mathrm{K} 25}$ ) 
displayed non-significant changes $(<2$-fold $)$ when comparing their spectral abundances in wt-neddylated and mt-neddylated $\sim$ Cul1-Rbx1 complexes (Table 1). Interestingly, the five interlinks of Cull (that is, Cul1 ${ }^{\mathrm{K} 337-\mathrm{K} 750}$, Cul1 ${ }^{\mathrm{K} 410-\mathrm{K} 750}$, $\mathrm{Cul1}{ }^{\mathrm{K} 464-\mathrm{K} 693}, \mathrm{Cull}^{\mathrm{K} 464-\mathrm{K} 743}$ and $\mathrm{Cul1}^{\mathrm{K} 693-\mathrm{K} 743}$ ) that were found primarily in Nedd8 Cul1-Rbx1 compared with unneddylated Cul1-Rbx1 were also difficult to detect in Nedd8(Q40E) Cul1-Rbx1 (Fig. 4a-e). Furthermore, the Cul1 ${ }^{\mathrm{K} 431-\mathrm{K} 472}$ interlink, which was found to be much more abundant in unneddylated Cul1-Rbx1 than Nedd8 Cul1-Rbx1, was also detected more intensely in Nedd8 (Q40E) Cul1-Rbx1 (Fig. 4f). Overall, the relative abundance ratios of these core CRL interlinks are similar when comparing Nedd8 $\sim$ Cul1-Rbx1 to both Cul1Rbx1 and Nedd8(Q40E) Cul1-Rbx1, respectively, suggesting that covalent attachment of Nedd8(Q40E) to Cul1 did not result in the same conformational changes in Cull as the wild-type Nedd8 modification.

This observation is also supported by the identification of interlinked peptides between Cull and Rbx1. The $\mathrm{Cull}^{\mathrm{K} 743}-\mathrm{Rbx}^{\mathrm{K} 89}$ and $\mathrm{Cul1}^{\mathrm{K} 750}-\mathrm{Rbx}^{\mathrm{K} 89}$ interprotein interlinks were primarily detected in Nedd8 Cull-Rbx1, but not in Cul1-Rbx1 or Nedd8(Q40E) Cul1-Rbx1 (Fig. 4g,h). Collectively, the quantitative MS profiles of the identified Nedd8(Q40E) Cul1-Rbx1 linkages are much more similar to those of unneddylated Cul1-Rbxl.

The structural dissimilarities in the two types of neddylated Cul1-Rbx1 complexes are further confirmed by Cul1-Nedd8 linkage comparisons. We have identified seven unique interprotein $\mathrm{K}-\mathrm{K}$ linkages between Cul1 and Nedd8 as summarized in Table 1. Interestingly, all of the Cul1-Nedd8 intersubunit interlinks had relative abundance ratios indicating significant differences ( $\geq 4$-fold) between Nedd8 Cul1-Rbx1 and Nedd8(Q40E) Cul1-Rbx1 (Fig. 5). Among them, five crosslinks $\left(\mathrm{Nedd} 8^{\mathrm{K} 6}{ }_{-} \mathrm{Cul} 1^{\mathrm{K} 410}\right.$, ${ }_{\mathrm{Nedd}} 8^{\mathrm{K} 6}{ }_{-\mathrm{Cul}} 1^{\mathrm{K} 464}$, Nedd $8^{\mathrm{K} 6}{ }_{-}$ $\mathrm{Cull}^{\mathrm{K} 468}$, Nedd $8^{\mathrm{K} 48}{ }_{-} \mathrm{Cul}_{1}{ }^{\mathrm{K} 693}$ and $\mathrm{Nedd} 8^{\mathrm{K} 6}{ }_{-\mathrm{Cul}}{ }^{\mathrm{K} 701}$ ) were only detected in Nedd $8 \sim \mathrm{Cul} 1-\mathrm{Rbx} 1$, while the remaining cross-links ( $\mathrm{Nedd}^{\mathrm{K} 6}{ }_{-\mathrm{Cul}}{ }^{\mathrm{K} 493}$ and $\mathrm{Nedd} 8^{\mathrm{K} 48}-\mathrm{Cul1}{ }^{\mathrm{K} 493}$ ) were only measured in Nedd8(Q40E) Cul1-Rbx1. In particular, K6 of Nedd8 and K493 of Cul1 are localized to opposite sides of the Cul1 scaffold in the Nedd8 $\sim$ Cul1-Rbx1 model, resulting in a $\mathrm{C}_{\alpha}-\mathrm{C}_{\alpha}$ distance $>30 \AA$. Therefore, this Nedd $8^{\mathrm{K} 6}-\mathrm{Cul1}^{\mathrm{K} 493}$ crosslink preferably detected in Nedd8(Q40E) C Cul1-Rbx1 further suggests that the Q40E mutation imparts a large degree of influence on the position of Nedd8 in relation to the Cull scaffold. Taken together, our results have demonstrated that Nedd8(Q40E) cannot induce the same structural effect on Cul1-Rbx1 as wild-type Nedd8, and the overall conformation of Nedd8(Q40E) Cul1-Rbx1 is much more similar to that of unneddylated Cul1-Rbxl (Fig. 5a-f).

\section{Discussion}

We have developed an effective QXL-MS workflow based on our previously developed pair of isotope-labelled (that is, $\mathrm{d}_{0}$ and $\mathrm{d}_{10}$ ) MS-cleavable DMDSSO ${ }^{31}$ to characterize the structural differences and similarities of three Cul1-Rbx1 complexes. This approach allows us to quantitatively assess neddylationdependent conformational changes within the Cul1-Rbx1 complex and gain insights into the molecular basis underlying its activation mechanism. In this work, we have demonstrated that DMDSSO reagents are well suited to quantitatively compare protein complexes as they cross-link proteins with similar efficiency and the relative spectral intensity ratios of $\mathrm{d}_{0^{-}}$and $\mathrm{d}_{10}$-DMDSSO cross-linked peptides are indicative of their respective abundances in the two compared samples. Thus, these isotope-coded cross-linkers can be used orthogonally to study differential protein structures by characterizing their intraprotein and interprotein interlinked peptides to describe protein interactions associated with conformational changes.

With this QXL-MS approach, we have reproducibly quantified 24 unique intraprotein and interprotein lysine-lysine linkages within the three different forms of Cul1-Rbx1 complexes (that is, Cul1-Rbx1, Nedd8 Cul1-Rbx1 and Nedd8(Q40E) Cul1Rbx1). Although a substantial amount of cross-link data correlates well with existing models, several cross-links have spatial distances outside the desired range and cannot be rationalized based on current structure models. While our results generally support the homology model of Nedd8 $\sim$ Cul1-Rbx1 derived from the Nedd8 $\sim \mathrm{Cul}^{\mathrm{CTD}}$-Rbx1 crystal structure ${ }^{14}$, a more accurate description of neddylation-induced conformational changes of the Cul1 scaffold calls for the necessity of a better defined model.

Independent of such a model, multiple pairwise comparisons have revealed that the molecular structure of Nedd8(Q40E)modified Cul1-Rbxl is very similar to that of its unmodified form, but significantly different from wild-type Nedd8-modified Cul1-Rbx1, indicating that Gln40 in Nedd8 is critical for the structural stability of neddylated Cul1-Rbx1. In the structure of Nedd8-Cul5 ${ }^{\mathrm{CTD}}$-Rbx1, Gln40 is proximal to the isopeptide bond between Nedd8 and Cul5 (ref. 14) and may interact with the cullin scaffold to stabilize its active conformation. On the other hand, the unmodified CRL catalytic core adopts a rigid, thermodynamically stable 'closed' structure, lacking ligase activity for polyubiquitination of substrates. During neddylation, CRL interacts with neddylation machinery and shifts to a flexible, 'open' conformation with the extending RINGfinger domain ${ }^{14,39}$. The neddylated CRL remains in its active state until Nedd8 is removed (deneddylation). We propose that Gln40 in Nedd8 can interact with amino-acid residues in cullin through weak interactions, such as hydrogen bonds and electrostatic interactions, which are responsible for stabilization of the 'open' state. Deamidation of Gln40 abolishes or weakens these interactions such that the CRL switches back to its thermodynamically more stable 'closed' state. On the basis of our cross-link data, we have proposed schematic models representing neddylation-dependent conformational changes in the Cul1-Rbx1 complex by wild-type or mutant Nedd8 (Fig. 5g). As illustrated, wt-neddylation leads to the 'open' conformation of the CRL core in which Rbx1 is free to rotate as previously shown by crystallography ${ }^{14}$. In contrast, mt-neddylation of Cul1 prevents switching from the inactive to active state by maintaining the 'closed' structure of the CRL with Rbx1 embedded. To verify this hypothesis, an experimental structure of neddylated full-length CRL will be required.

In summary, we have successfully applied our recently developed MS-cleavable, stable isotope-labelled cross-linkers $\mathrm{d}_{0^{-}}$ DMDSSO and $\mathrm{d}_{10}$-DMDSSO to quantitatively study structural differences in Cul1-Rbx1 complexes in response to neddylation. Such structural characterization has previously been hindered using conventional structural tools because of their large sizes (over $100 \mathrm{kDa}$ ) and dynamic conformations. Our QXL-MS approach enables us to quantitatively compare multiple lysine interlinks in three types of full-length Cul1-Rbxl complexes. Comparing these cross-linkage profiles, we found that neddylation can induce large structural rearrangements of the Cul1-Rbx1 complex, which are partially consistent with structural models obtained with truncated and neddylated Cul5-Rbxl complex. Our results also indicates Nedd8(Q40E)-conjugated Cul1-Rbx1 has a similar structure as that of free Cul1-Rbxl, answering the puzzle of how a subtle change of a single Nedd8 amino acid, $\mathrm{Gln}^{40}$, can abolish the activity of the much larger CRL complex. Given the speed and accuracy of the approach, we expect that our 

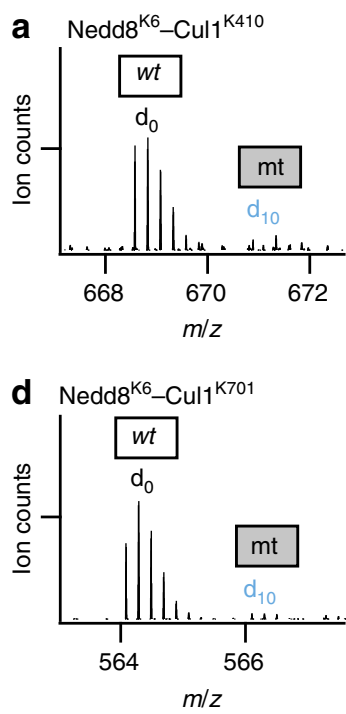

$w t:$ Nedd8 Cul1-Rbx1
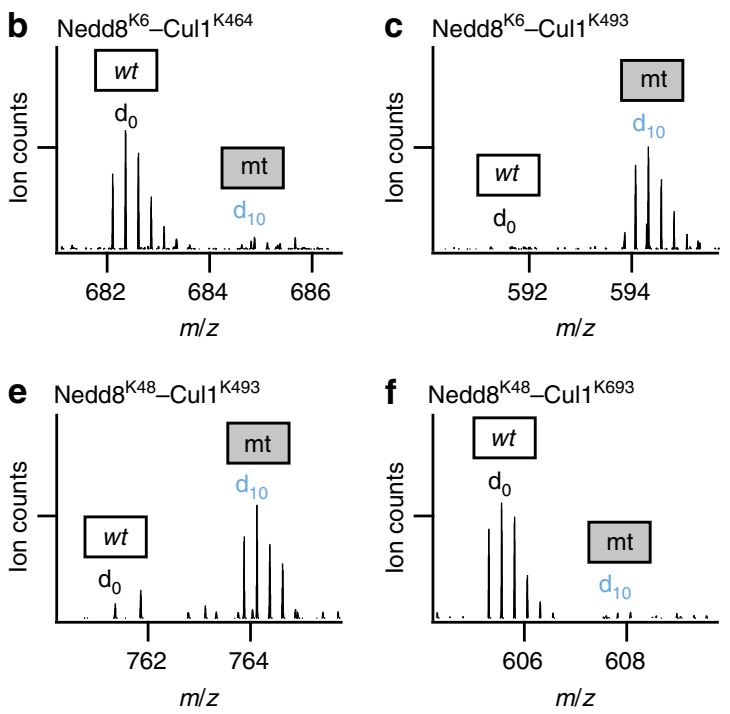

$\mathrm{mt}:$ Nedd8(Q40E) Cul1-Rbx1

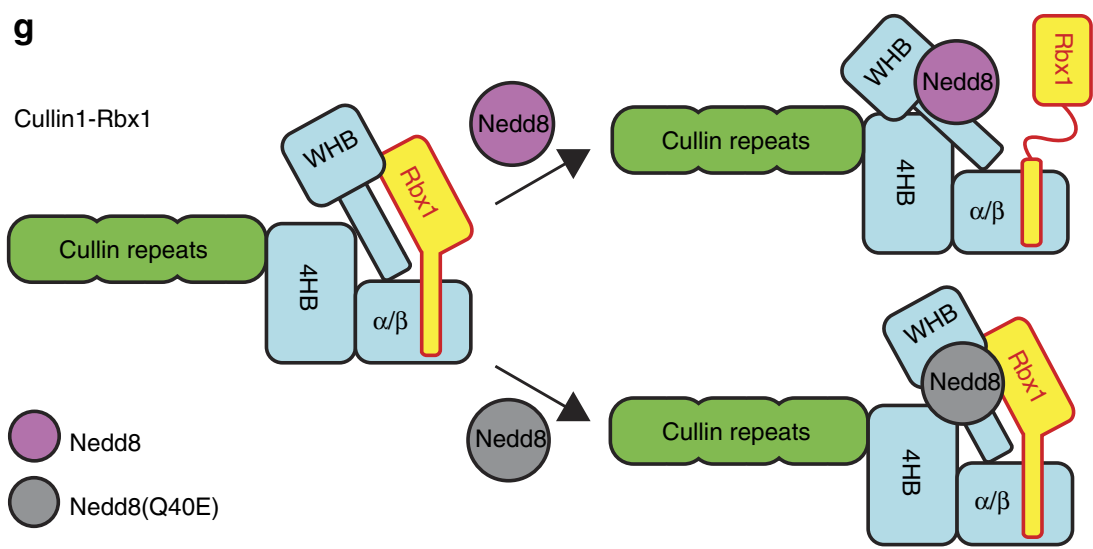

Figure 5 | Elucidation of structural dissimilarities between wt- and mt-neddylated Cul1-Rbx1 complexes by quantitative analysis of Cul1-Nedd8 interlinks. MS1 spectra of (a) Nedd8 $8^{\mathrm{K} 6}-\mathrm{Cul} 1^{\mathrm{K} 410}$, (b) Nedd8 $8^{\mathrm{K} 6}-\mathrm{Cul} 1^{\mathrm{K} 464}$, (c) Nedd8 $8^{\mathrm{K} 6}-\mathrm{Cul} 1^{\mathrm{K} 493}$, (d) Nedd8 $8^{\mathrm{K} 6}-\mathrm{Cul} 1^{\mathrm{K} 701}$, (e) $\mathrm{Nedd} 8^{\mathrm{K} 48}-\mathrm{Cul1}{ }^{\mathrm{K} 493}$ and (f) Nedd8 ${ }^{\mathrm{K} 48}$-Cul1 ${ }^{\mathrm{K} 693}$. The $\mathrm{mt}$ form was $\mathrm{d}_{0}$-DMDSSO cross-linked, and the wt form was $\mathrm{d}_{10}$-DMDSSO cross-linked. (g) Proposed models for Nedd8dependent conformational changes of the Cul1-Rbx1 complex.

QXL-MS strategy will enable us to perform future studies in characterizing E2-E3 interactions and further dissect the action mechanism of CRLs during protein ubiquitination. In addition, our work has paved the way for adapting QXL-MS methods for elucidating dynamic structures of proteins and protein complexes in the future.

\section{Methods}

Materials and reagents. General chemicals were purchased from Fisher Scientific or VWR international. Sequencing grade-modified trypsin was purchased from Promega (Fitchburg, WI).

Preparation of Cul1-Rbx1 protein complexes. The heterodimeric NEDD8-activating enzyme APPBP1-Uba3 was prepared similarly as before ${ }^{40}$. Briefly, APPBP1 was subcloned into a modified pGEX4T1 (Amersham Biosciences) vector containing a glutathione $S$-transferase (GST) tag followed by a Tobacco etch virus (TEV) protease cleavage site, while Uba3 was subcloned into a modified pET15b (Novagen) vector containing a chloramphenicol resistance cassette. GST-APPBP1 and Uba3 were co-expressed in BL21(DE3) (Novagen) and purified by glutathioneaffinity chromatography. After TEV cleavage, the APPBP1-Uba3 complex was further purified by anion exchange and gel filtration.

Nedd 8 and the Nedd8-conjugating enzyme Ubc12 were subcloned into the same pGEX4T1 vector. Both were expressed in E. coli BL-21(DE3) cells and purified by glutathione-affinity and anion-exchange chromatography. In this study, we used a truncated version of $\mathrm{Nedd} 8$ ending at glycine 76 , representing its mature form.
Two short unstructured segments in the N-terminus of Cul1 (residues 1-12 and 58-81) were removed from full-length human Cull to form Cul1 ${ }^{\Delta \mathrm{N}}$ (referred to here as Cul1). Both Cull and Rbx1 ${ }^{16-108}$ were fused with an N-terminal $\mathrm{His}_{6}$ tag followed by a TEV cleavage site and co-expressed in BL-21(DE3). The complex was first purified by a $\mathrm{Ni}^{2}+$ sepharose-affinity column (GE Healthcare) and further purified by cation-exchange and gel filtration chromatography after TEV cleavage. To prepare Nedd $8 \sim$ Cul1-Rbx1, $10 \mu \mathrm{M}$ purified Cul1-Rbx1 was neddylated with $10 \mu \mathrm{M}$ GST-Nedd 8 in the presence of $0.2 \mu \mathrm{M}$ APPBP1-Uba3 and $0.5 \mu \mathrm{M}$ Ubc12 for $1 \mathrm{~h}$ at $4{ }^{\circ} \mathrm{C}$. Nedd $8 \sim \mathrm{Cull}-\mathrm{Rbx} 1$ was then separated from free Cull-Rbxl by a glutathione-affinity column. After TEV cleavage, Nedd $8 \sim \mathrm{Cul1-Rbx1}$ was eluted off the column and further purified by cation-exchange and gel filtration chromatography. Nedd8(Q40E)-modified Cul1-Rbx1 was purified similarly to the wild type.

Full-length human ubiquitin-activating enzyme Ubel was expressed as a GST fusion protein in High Five insect cells using the Bac-to-Bac baculovirus expression system (Invitrogen). Insect cells were collected $48-72 \mathrm{~h}$ post infection and lysed, followed by glutathione-affinity chromatography. Recombinant human Cdc34 was overexpressed and purified from $E$. coli by a similar approach as the Nedd8 purification. Recombinant untagged ubiquitin (Ub) was expressed in BL21(DE3). After sonication and centrifugation, cleared lysate was adjusted to $3.5 \%$ perchloric acid. After precipitated protein was removed by centrifugation, ubiquitin in the supernatant was further purified by cation-exchange chromatography and dialysis against $20 \mathrm{mM}$ Tris, $\mathrm{pH} 8.0$ thoroughly.

Ubiquitination assays. For free ubiquitin chain synthesis assay, a mixture containing $100 \mu \mathrm{M}$ Ub, $0.3 \mu \mathrm{M}$ UBE1 and $1.0 \mu \mathrm{M}$ Cdc34 was incubated with $0.4 \mu \mathrm{M}$ Cul1-Rbxl variants in a reaction buffer of $50 \mathrm{mM}$ Tris- $\mathrm{HCl}, 200 \mathrm{mM} \mathrm{NaCl}$, $2 \mathrm{mM}$ ATP and $10 \mathrm{mM} \mathrm{MgCl}_{2}, \mathrm{pH} 8.0$. After incubation at $37^{\circ} \mathrm{C}$ for $4 \mathrm{~h}$, the 
reaction mixtures were resolved by a $15 \%$ SDS-PAGE gel and transferred onto a nitrocellulose membrane, which was incubated overnight with a mouse monoclonal anti-ubiquitin antibody (Sigma-Aldrich, \#U0508) at 1:2,500 dilution. The membrane was washed and incubated with horseradish peroxidase-linked ECL-anti mouse IgG (GE Healthcare, \#NA931V) for $1 \mathrm{~h}$. Free ubiquitin chains were visualized using SuperSignal West Pico Chemiluminescent Substrate (Pierce Biotechnology, \#34080).

For the CRY2 ubiquitination assay, a reaction mixture containing $0.2 \mu \mathrm{M}$ CRY2-FBXL3-SKP1 complex ${ }^{36}, 70 \mu \mathrm{M}$ Ub, $0.15 \mu \mathrm{M} \mathrm{UBE} 1$ and $1.5 \mu \mathrm{M}$ Cdc34 was incubated with $0.4 \mu \mathrm{M}$ Cull-Rbxl variants in a reaction buffer of $40 \mathrm{mM}$ Tris-HCl (pH 7.5), $2 \mathrm{mM}$ dithiothreitol, $5 \mathrm{mM} \mathrm{MgCl}_{2}$ and $2 \mathrm{mM}$ ATP. The reactions were carried out at $37^{\circ} \mathrm{C}$ and quenched at different time points by adding SDS-PAGE loading buffer, then analysed by western blot with a rabbit anti-CRY2 antibody(LifeSpan BioSciences, Inc. \#LS-C6229) at 1:1,000 dilution and horseradish peroxidase-linked ECL-anti rabbit IgG (GE Healthcare, \#NA934V).

\section{DMDSSO cross-linking and digestion of Cul1-Rbx1 complexes. Purified} complexes were diluted to $4 \mu \mathrm{M}$ in $20 \mathrm{mM}$ HEPES ( $\mathrm{pH} 7.5$ ) and reacted with $\mathrm{d}_{0^{-}}$or $\mathrm{d}_{10}$-DMDSSO in a molar ratio of 1:25 (protein: cross-linker) for $45 \mathrm{~min}$ at room temperature and quenched with excess ammonium bicarbonate. Cross-linked proteins were then separated by SDS-PAGE and visualized by Coomassie blue. Bands corresponding to cross-linked complexes were excised, reduced with tris (2-carboxyethyl) phosphine for $30 \mathrm{~min}$ at room temperature and alkylated with chloroacetamide for $30 \mathrm{~min}$ at room

temperature in dark, and then digested with trypsin at $37^{\circ} \mathrm{C}$ overnight. Peptide digests were extracted, concentrated and reconstituted in 3\% ACN/2\% formic acid before LC-MS ${ }^{n}$ analysis. To allow quantitative pairwise complex comparisons, individually cross-linked proteins were strategically mixed, for example, $\mathrm{d}_{0}$-DMDSSO cross-linked Cul1-Rbx1 with $\mathrm{d}_{10}$-DMDSSO cross-linked Nedd8 Cul1-Rbx1, at a 1:1 ratio and subjected to subsequent analysis together as outlined above.

Liquid chromatography-multistage tandem mass spectrometry. DMDSSO cross-linked peptides were analysed by LC-MS ${ }^{n}$ utilizing an LTQ-Orbitrap XL MS (Thermo Fisher, San Jose, CA) coupled on-line with an Easy-nLC 1,000 (Thermo Fisher, San Jose, CA) as previously described ${ }^{29,31}$. Each MS $^{n}$ experiment consists of one MS scan in FT mode $(350-1,400 \mathrm{~m} / \mathrm{z}$, resolution of 60,000 at $\mathrm{m} / \mathrm{z} 400$ ) followed by two data-dependent MS2 scans in FT mode (resolution of 7,500) with normalized collision energy at $20 \%$ on the top two MS peaks with charges at $3+$ or up, and three MS3 scans in the LTQ with normalized collision energy at 35\% on the top three peaks from each MS2.

\section{Data analysis, identification and quantification of cross-linked peptides}

Monoisotopic masses of parent ions and corresponding fragment ions, parent ion charge states, and ion intensities from LC-MS ${ }^{2}$ and LC-MS ${ }^{3}$ spectra were extracted using an in-house software based on the Raw Extract script from Xcalibur v2.4 (Thermo Scientific) $^{29-31}$. MS $^{3}$ data were subjected to a developmental version of Protein Prospector (v. 5.10.10) for database searching, using Batch-Tag against a limited database containing recombinant Cul1, Rbx1, Nedd8 and Nedd8(Q40E) sequences with mass tolerances for parent ions and fragment ions set as \pm 20 p.p.m. and $0.6 \mathrm{Da}$, respectively. Trypsin was set as the enzyme with five maximum missed cleavages allowed. A maximum of five variable modifications were also allowed, including protein $\mathrm{N}$-terminal acetylation, methionine oxidation, $\mathrm{N}$-terminal conversion of glutamine to pyroglutamic acid, asparagine deamidation and cysteine carbamidomethylation. In addition, three defined modifications on uncleaved lysines and free protein $\mathrm{N}$-termini were also selected: alkene $\left(\mathrm{A}: \mathrm{C}_{4} \mathrm{H}_{4} \mathrm{O}\right.$, $+68 \mathrm{Da}$; or $\left.\mathrm{A}^{*}: \mathrm{C}_{4} \mathrm{H}_{-1} \mathrm{D}_{5} \mathrm{O},+73 \mathrm{Da}\right)$, sulfenic acid $\left(\mathrm{S}: \mathrm{C}_{4} \mathrm{H}_{6} \mathrm{O}_{2} \mathrm{~S},+118 \mathrm{Da}\right.$; or $\mathrm{S}^{*}$ : $\left.\mathrm{C}_{4} \mathrm{H}_{1} \mathrm{D}_{5} \mathrm{O}_{2} \mathrm{~S},+123 \mathrm{Da}\right)$ and unsaturated thiol $\left(\mathrm{T}: \mathrm{C}_{4} \mathrm{H}_{4} \mathrm{OS},+100 \mathrm{Da}\right.$; or $\mathrm{T}^{*}$ : $\mathrm{C}_{4} \mathrm{H}_{-1} \mathrm{D}_{5} \mathrm{OS},+105 \mathrm{Da}$ ) modifications, due to remnant moieties of $\mathrm{d}_{0^{-}}$(that is, $\mathrm{A}$, $S$ and $T$ ) or $d_{10}$-DMDSSO (that is, $A^{\star}, S^{*}$ and $T^{*}$ ), respectively. It is noted that the sulfenic acid moiety often undergoes dehydration to become a more stable and dominant unsaturated thiol moiety (that is, $\mathrm{T},+100 \mathrm{Da}$ or $\mathrm{T}^{\star},+105 \mathrm{Da}$ ) as previously described ${ }^{29-31}$. Initial acceptance criteria for peptide identification required a reported expectation value $\leq 0.1$.

Integration of $\mathrm{MS}^{n}$ data was carried out using the in-house program LinkHunter, a revised version of the previously written Link-Finder program, to validate and summarize cross-linked peptides ${ }^{22,29}$. Basically, monoisotopic masses of parent ions measured in $\mathrm{MS}^{1}$ scans for those putative interlinked peptides are required to match the sum of the two $\mathrm{MS}^{2}$ cross-linked fragment ions that have been sequenced in $\mathrm{MS}^{3}$.

Only the identified interlinked DMDSSO cross-linked peptides were subjected for subsequent manual quantitation as only interlinked peptides provide the most useful information on protein structures. Using Skyline (v. 2.5.06157; https://skyline.gs.washington.edu), we have determined the spectral abundances of all individually identified cross-linked peptides in each pairwise comparison, and the calculated relative abundance of $\mathrm{d}_{0} / \mathrm{d}_{10}$ cross-linked peptides. This allows determining the relative occurrence of the identified $\mathrm{K}-\mathrm{K}$ linkages across all purified complexes. All linkages were then mapped onto existing Cul1-Rbx1 crystal structure (PDB: 1LDJ), as well as the derived homology model of Nedd8 $\sim$ Cul1-Rbx1 (ref. 32), to compare experimentally derived ratios of occurrence of $\mathrm{K}-\mathrm{K}$ linkages to the $\mathrm{C}_{\alpha}-\mathrm{C}_{\alpha}$ distances as determined by structural models.

\section{References}

1. Fang, S., Lorick, K. L., Jensen, J. P. \& Weissman, A. M. RING finger ubiquitin protein ligases: implications for tumorigenesis, metastasis and for molecular targets in cancer. Semin. Cancer Biol. 13, 5-14 (2003).

2. Petroski, M. D. \& Deshaies, R. J. Function and regulation of cullin-RING ubiquitin ligases. Nat. Rev. Mol. Cell Biol. 6, 9-20 (2005).

3. Nalepa, G., Rolfe, M. \& Harper, J. W. Drug discovery in the ubiquitinproteasome system. Nat. Rev. Drug Discov. 5, 596-613 (2006).

4. Zimmerman, E. S., Schulman, B. A. \& Zheng, N. Structural assembly of cullin-RING ubiquitin ligase complexes. Curr. Opin. Struct. Biol. 20, 714-721 (2010).

5. Sarikas, A., Hartmann, T. \& Pan, Z. Q. The cullin protein family. Genome Biol. 12, 220 (2011).

6. Duda, D. M. et al. Structural regulation of cullin-RING ubiquitin ligase complexes. Curr. Opin. Struct. Biol. 21, 257-264 (2011)

7. Deshaies, R. J. SCF and Cullin/Ring H2-based ubiquitin ligases. Annu. Rev. Cell Dev. Biol. 15, 435-467 (1999).

8. Cardozo, T. \& Pagano, M. The SCF ubiquitin ligase: insights into a molecular machine. Nat. Rev. Mol. Cell Biol. 5, 739-751 (2004).

9. Zheng, N., Wang, P., Jeffrey, P. D. \& Pavletich, N. P. Structure of a c-CblUbcH7 complex: RING domain function in ubiquitin-protein ligases. Cell 102, 533-539 (2000).

10. Podust, V. N. et al. A Nedd8 conjugation pathway is essential for proteolytic targeting of p27Kip1 by ubiquitination. Proc. Natl Acad. Sci. USA 97, 4579-4584 (2000).

11. Read, M. A. et al. Nedd8 modification of cul-1 activates SCF(beta(TrCP))dependent ubiquitination of IkappaBalpha. Mol. Cell. Biol. 20, 2326-2333 (2000).

12. Amir, R. E., Iwai, K. \& Ciechanover, A. The NEDD8 pathway is essential for SCF(beta - TrCP)-mediated ubiquitination and processing of the NF-kappa B precursor p105. J. Biol. Chem. 277, 23253-23259 (2002).

13. Saha, A. \& Deshaies, R. J. Multimodal activation of the ubiquitin ligase SCF by Nedd8 conjugation. Mol. Cell 32, 21-31 (2008).

14. Duda, D. M. et al. Structural insights into NEDD8 activation of cullin-RING ligases: conformational control of conjugation. Cell 134, 995-1006 (2008).

15. Cui, J. et al. Glutamine deamidation and dysfunction of ubiquitin/NEDD8 induced by a bacterial effector family. Science 329, 1215-1218 (2010).

16. Jubelin, G. et al. Pathogenic bacteria target NEDD8-conjugated cullins to hijack host-cell signaling pathways. PLoS Pathog. 6, e1001128 (2010).

17. Morikawa, H. et al. The bacterial effector Cif interferes with SCF ubiquitin ligase function by inhibiting deneddylation of Cullin1. Biochem. Biophys. Res. Commun. 401, 268-274 (2010).

18. Toro, T. B., Toth, J. I. \& Petroski, M. D. The cyclomodulin cycle inhibiting factor (CIF) alters cullin neddylation dynamics. J. Biol. Chem. 288, 14716-14726 (2013)

19. Leitner, A. et al. Probing native protein structures by chemical cross-linking, mass spectrometry, and bioinformatics. Mol. Cell. Proteomics 9, 1634-1649 (2010).

20. Chen, Z. A. et al. Architecture of the RNA polymerase II-TFIIF complex revealed by cross-linking and mass spectrometry. EMBO J. 29, 717-726 (2010).

21. Herzog, F. et al. Structural probing of a protein phosphatase $2 \mathrm{~A}$ network by chemical cross-linking and mass spectrometry. Science 337, 1348-1352 (2012).

22. Kao, A. et al. Mapping the structural topology of the yeast $19 \mathrm{~S}$ proteasomal regulatory particle using chemical cross-linking and probabilistic modeling. Mol. Cell. Proteomics 11, 1566-1577 (2012).

23. Leitner, A. et al. The molecular architecture of the eukaryotic chaperonin TRiC/ CCT. Structure 20, 814-825 (2012).

24. Schmidt, C. et al. Comparative cross-linking and mass spectrometry of an intact F-type ATPase suggest a role for phosphorylation. Nat. Commun. 4, 1985 (2013).

25. Erzberger, J. P. et al. Molecular architecture of the 40SeIF1eIF3 translation initiation complex. Cell 158, 1123-1135 (2014)

26. Shi, Y. et al. Structural characterization by cross-linking reveals the detailed architecture of a coatomer-related heptameric module from the nuclear pore complex. Mol. Cell. Proteomics 13, 2927-2943 (2014).

27. Lasker, K. et al. Molecular architecture of the $26 \mathrm{~S}$ proteasome holocomplex determined by an integrative approach. Proc. Natl Acad. Sci. USA 109, 1380-1387 (2012).

28. Zeng-Elmore, X. et al. Molecular architecture of photoreceptor phosphodiesterase elucidated by chemical cross-linking and integrative modeling. J. Mol. Biol. 426, 3713-3728 (2014). 
29. Kao, A. et al. Development of a novel cross-linking strategy for fast and accurate identification of cross-linked peptides of protein complexes. Mol. Cell. Proteomics 10, M110.002212 (2011).

30. Kaake, R. M. et al. A new in vivo cross-linking mass spectrometry platform to define protein-protein interactions in living cells. Mol. Cell. Proteomics 13, 3533-3543 (2014)

31. Yu, C., Kandur, W., Kao, A., Rychnovsky, S. \& Huang, L. Developing new isotope-coded mass spectrometry-cleavable cross-linkers for elucidating protein structures. Anal. Chem. 86, 2099-2106 (2014).

32. Zheng, N. et al. Structure of the Cul1-Rbx1-Skp1-F boxSkp2 SCF ubiquitin ligase complex. Nature 416, 703-709 (2002).

33. Busino, L. et al. SCFFbxl3 controls the oscillation of the circadian clock by directing the degradation of cryptochrome proteins. Science 316, 900-904 (2007).

34. Godinho, S. I. et al. The after-hours mutant reveals a role for Fbxl3 in determining mammalian circadian period. Science 316, 897-900 (2007).

35. Siepka, S. M. et al. Circadian mutant Overtime reveals F-box protein FBXL3 regulation of cryptochrome and period gene expression. Cell 129, 1011-1023 (2007).

36. Xing, W. et al. SCF(FBXL3) ubiquitin ligase targets cryptochromes at their cofactor pocket. Nature 496, 64-68 (2013)

37. Wu, K., Chen, A. \& Pan, Z. Q. Conjugation of Nedd8 to CUL1 enhances the ability of the ROC1-CUL1 complex to promote ubiquitin polymerization. $J$. Biol. Chem. 275, 32317-32324 (2000)

38. Schmidt, C. \& Robinson, C. V. A comparative cross-linking strategy to probe conformational changes in protein complexes. Nat. Protoc. 9, 2224-2236 (2014).

39. Scott, D. C. et al. Structure of a RING E3 trapped in action reveals ligation mechanism for the ubiquitin-like protein NEDD8. Cell 157, 1671-1684 (2014).

40. Huang, D. T. \& Schulman, B. A. Expression, purification, and characterization of the E1 for human NEDD8, the heterodimeric APPBP1-UBA3 complex. Methods Enzymol. 398, 9-20 (2005).

\section{Acknowledgements}

We wish to thank members of the Huang and Zheng laboratories for their help during this study, especially Alex Huszagh in the Huang lab for his help in data analysis. We would like to thank Professor A.L. Burlingame, Drs Robert Chalkley, Shenheng Guan and Peter Baker at UCSF for using Protein Prospector. This work was supported by National Institutes of Health grants RO1GM074830 to L.H. and R01GM106003 to L.H. and S.R N.Z. is an Howard Hughes Medical Institute investigator. Eric Novitsky was supported by an institutional Chemical and Structural Biology Training Grant predoctoral fellowship (T32-GM10856).

\section{Author contributions}

C.Y. designed and carried out QXL-MS experiments and data analyses, and prepared all figures and tables. H.M. prepared purified protein samples and performed ubiquitination assay. X.T. contributed to ubiquitination assay. E.J.N. and S.D.R. synthesized crosslinking reagents; L.H. and N.Z. conceived the study and directed the research. C.Y., H.M., N.Z. and L.H. wrote the paper.

\section{Additional information}

Supplementary Information accompanies this paper at http://www.nature.com/ naturecommunications

Competing financial interests: The authors declare no competing financial interests.

Reprints and permission information is available online at http://npg.nature.com/ reprintsandpermissions/

How to cite this article: Yu, C. et al. Gln40 deamidation blocks structural reconfiguration and activation of SCF ubiquitin ligase complex by Nedd8. Nat. Commun 6:10053 doi: 10.1038/ncomms10053 (2015).

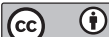

This work is licensed under a Creative Commons Attribution 4.0 International License. The images or other third party material in this article are included in the article's Creative Commons license, unless indicated otherwise in the credit line; if the material is not included under the Creative Commons license, users will need to obtain permission from the license holder to reproduce the material. To view a copy of this license, visit http://creativecommons.org/licenses/by/4.0/ 